\title{
The Evidential Reasoning Approach to Medical Diagnosis using Intuitionistic Fuzzy Dempster-Shafer Theory*
}

\author{
Yanni Wang ${ }^{1}$, Yaping Dai ${ }^{2}$ Yu-wang Chen ${ }^{3}$ Fancheng Meng ${ }^{4}$ \\ ${ }^{1}$ School of Automation, Beijing Institute of Technology, \\ Zhongguancun street, \\ Beijing, Haidian District, P. R. China \\ E-mail:wangni_1983@163.com \\ ${ }^{2}$ School of Automation, Beijing Institute of Technology, \\ Zhongguancun south street 5 , \\ Beijing, Haidian District, P. R. China \\ E-mail: daiyaping@bit.edu.cn \\ ${ }^{3}$ Decision and Cognitive Sciences Research Centre, Manchester Business School, \\ Manchester, M15 6PB, United Kingdom \\ E-mail:Yu-wang.Chen@mbs.ac.uk \\ www. manchester.ac.uk \\ ${ }^{4}$ School of Automation, Beijing Institute of Technology, \\ Zhongguancun south street 5 , \\ Beijing, Haidian District, P. R. China \\ E-mail:sdmfwzh@163.com
}

Received 9 March 2014

Accepted 17 June 2014

\begin{abstract}
For medical diagnosis, fuzzy Dempster-Shafer theory is extended to model domain knowledge under probabilistic and fuzzy uncertainty. However, there are some information loss using discrete fuzzy sets and traditional matching degree method. This study aims to provide a new evidential structure to reduce information loss. This paper proposes a new intuitionistic fuzzy evidential reasoning (IFER) approach which combines intuitionistic trapezoidal fuzzy numbers and inclusion measure to improve the accuracy of representation and reasoning. The proposed approach has been validated by a stroke diagnosis. It is shown that the IFER approach leads to more accurate results.
\end{abstract}

Keywords: Fuzzy sets, evidential reasoning, uncertainty, Dempster-Shafer theory, inclusion measure, medical diagnosis.

\footnotetext{
* Supported by Program for Changjiang Scholars and Innovative Research Team in University under Grant IRT1208. Guided and revised by Prof. Jian-bo Yang, Prof. Jie Lu and Prof. Witold Pedrycz.

${ }^{\dagger}$ Corresponding author. E-mail: wangni_1983@163.com.Academy of Control Science and Engineering. Her main research interests include decision making, fuzzy logic, and optimal methodology.

${ }^{\ddagger}$ Zhongguancun south street 5, Beijing, Haidian District,100190, P. R. China.
} 


\section{Introduction}

A key component of medical diagnosis is concerned with the representation and reasoning of medical domain knowledge. Most medical knowledge can be structured in the form of IF-THEN rules. In each rule, we distinguish antecedent attributes (causes) and consequents (effects) e.g. (Ref. 1). For instance, an IF-THEN rule for stroke diagnosis may involve 4 stroke symptoms and 3 consequents. However, stroke symptoms are often described by stoker using linguistic terms. Hence the attributes of initial IF-THEN rules provided by medical expert usually involve linguistic variables. Kong e.g. (Ref. 2) discussed that uncertainty is mostly resulting from subjective domain knowledge or various clinical symptoms. In addition, considering the probability of occurrence of attributes is not always 1 , belief degree is taken into account in IF-THEN rules. Lin e.g. (Ref. 3)stated that the result of medical diagnosis is mainly determined by handling various types of uncertainty. In order to complete medical diagnosis, we refer to various frameworks for representing and reasoning for medical domain knowledge under probabilistic and fuzzy uncertainty: multiple attribute decision analysis (MADA), fuzzy set (FS) theory and evidential reasoning (ER). There is a tendency that applying the latest development in these three aspects to medical diagnosis. Evidence theory as a generalization of possibility theory is also known as Dempster-Shafer theory. Jones e.g. (Ref. 4) built a framework for medical diagnosis using the evidence theory. Durbach and Stewart e.g. (Ref. 5) noted that the DS theory of evidence reasoning can deal with probabilistic uncertainty by replacing subjective probabilities with degrees of belief. To model two types of uncertainty, namely fuzziness and probability, DS theory of evidence is extended into fuzzy DS (FDS) theory during the recent years. Xu e.g. (Ref. 6) made a review of the evidential reasoning (ER) approach about its theoretical development and applications. As such, there is a trend that the DS theory of evidence reasoning can be extended to FDS scheme e.g.( (Ref. 7), (Ref. 8) and (Ref. 9)). FDS evidence reasoning algorithm can be divided into three steps e.g. (Ref. 10): building a fuzzy evidence structure, combining evidence, and decision making based on ranking consequences.

Uncertainty of evidence is mainly reflected in the following aspects: attribute assessment, attribute weights, rule weights. During the development of the FDS theory, uncertain evidence can be expressed by fuzzy sets, interval numbers, and fuzzy numbers. Yang e.g. (Ref. 11) proposed a rule-based inference methodology using the evidential reasoning (RIMER) approach by adding belief degree of inputs and outputs into the traditional IF-THEN rule base. During the process of building a fuzzy evidence structure, the nonlinear relationship between antecedent attributes and consequents can be established. Max-min operation is adopted to set the matching degree between fuzzy sets in transformation of inputs. Sevastianov e.g. (Ref. 12) discussed that there are two restrictions in the RIMER approach. One of them is that the RIMER approach did not provide the combination method of different evidence. Basically, the ER approach made use of Dempster's rule of combination to aggregate attributes e.g. (Ref. 13).When the evidence structure was expressed by interval evidence, existing combination methods often lead to irrational structure because of improper treatment for the normalization process. Wang e.g. (Ref. 14) analyzed the interval data operations and presented the nonlinear combination method of interval belief degrees. Guo et al. e.g. (Ref. 15) handled the interval beliefs and interval weights to develop an enhanced ER approach. Aminravan et al. e.g. (Ref. 16) developed both fuzzy interval-grade and interval-valued belief degree (IGIB) using one of generalized fuzzy sets (i.e., vague sets). Max-min operation is adopted to set the matching degree between vague sets during the transformation of inputs. Husain e.g. (Ref. 17) concluded that the concept of intuitionistic fuzzy sets (IFSs) can be viewed as another generalized fuzzy set in cases where a conventional fuzzy set is not sufficient for definition of imprecise information. An interpretation of IFSs in terms of evidence theory was presented through converting IFSs into interval fuzzy sets (IVFSs) e.g. (Ref. 18). The operations on IFSs were presented and interval comparison methods were also discussed in the frame- 
work of the DS theory of evidence e.g. (Ref. 19). Wang e.g. (Ref. 20) applied triangular intuitionistic fuzzy numbers to fuzzy evidence reasoning. Hence, IFSs can be used to represent those nonspecific attributes. However, in most cases input attributes and antecedent attributes form pieces of linguistic knowledge. Using fuzzy numbers we can represent uncertainty to the highest extent than when dealing with discrete fuzzy sets. Furthermore, max-min operation between fuzzy sets may lead to significant losses of information. In particular, the extreme values representing the dominating attributes are only taken into account in this operation e.g. (Ref. 21). The values in-between are always neglected by decision makers. In order to improve the accuracy of the decision results, it is necessary to ensure the minimal losses of information.

The objective of the intuitionistic fuzzy evidential reasoning (IFER) approach is to offer representation and reasoning on a basis of linguistic knowledge under uncertainty more accurately by rebuilding the evidential structure so as to reduce information losses. In essence, it is to extend the basic RIMER methodology. The main improvement in the proposed IFER approach involves two aspects: on the one hand, the representation of knowledge uses continuous fuzzy numbers (intuitionistic trapezoidal fuzzy numbers, ITFN) instead of conventional discrete fuzzy sets. On the other hand, the matching degree method uses inclusion measure instead of maxmin operation for avoiding the effect by extreme values.

This paper is organized as follows: In Section 2, basic concept and existing evidence structure are reviewed. In Section 3, in order to reduce information loss in existing evidence structure, we propose a new intuitionistic fuzzy evidential reasoning (IFER) approach by rebuilding evidence structure. Firstly, intuitionistic fuzzy numbers are converted into interval fuzzy numbers by $\alpha$-cuts. Secondly, the belief degrees of consequents are updated according to the matching degree between inputs and antecedent attributes. Thirdly, attribute weights are calculated by normalizing the certainty factor. Fourthly, after interval belief degrees and weights are calculated, a nonlinear model is built to combine interval belief degrees. At last, the utility function is adopted to rank the combined results. In Section 4, a stroke diagnosis is presented to illustrate the validity and applicability of the proposed IFER method. In Section 5 , conclusions and the further study are summarized.

\section{Preliminaries}

In this section, basic concepts and former research achievements of intuitionisitc fuzzy set theory and evidential reasoning theory are introduced.

\subsection{Basic concept of intuitionistic trapezoidal fuzzy numbers}

Definition 1 Let $A$ be an intuitionistic trapezoidal fuzzy number (ITFN) defined in the space of real numbers $R$, its membership function is given by e.g. (Ref. 22)

$$
\mu_{A}(x)=\left\{\begin{array}{rr}
0, & x<a_{1}, \\
\frac{x-a_{1}}{a_{2}-a_{1}}, & a_{1} \leqslant x \leqslant a_{2}, \\
1, & a_{2} \leqslant x \leqslant a_{3}, \\
\frac{x-a_{3}}{a_{4}-a_{3}}, & a_{3} \leqslant x \leqslant a_{4}, \\
0, & a_{4}<x
\end{array}\right.
$$

And its non-membership function is given by

$$
v_{A}(x)=\left\{\begin{array}{rr}
1, & x<b_{1}, \\
\frac{x-b_{2}}{b_{1}-b_{2}}, & b_{1} \leqslant x \leqslant b_{2}, \\
0, & b_{2} \leqslant x \leqslant b_{3}, \\
\frac{x-b_{3}}{b_{4}-b_{3}}, & b_{3} \leqslant x \leqslant b_{4}, \\
1, & b_{4}<x
\end{array}\right.
$$

with the parameters $a_{1}, a_{2}, a_{3}, a_{4}, b_{1}, b_{2}, b_{3}, b_{4} \in$ $R, \quad b_{1} \leqslant a_{1} \leqslant b_{2} \leqslant a_{2} \leqslant a_{3} \leqslant b_{3} \leqslant a_{4} \leqslant b_{4}$. ITFN is then denoted as $A=\left\langle\left(a_{1}, a_{2}, a_{3}, a_{4}\right),\left(b_{1}, b_{2}, b_{3}, b_{4}\right)\right\rangle$. A useful tool to deal with the intuitionistic fuzzy numbers is using $\alpha$-cuts.Every $\alpha$-cut is a closed in- 
terval. They are calculated as follows e.g. (Ref. 23)

$$
\begin{aligned}
A_{\text {Low }}^{+}(\alpha) & =\inf \left\{x \in R \mid \mu_{A}(x) \geqslant \alpha\right\} \\
& =\left(a_{2}-a_{1}\right) \alpha+a_{1}, \\
A_{U p}^{+}(\alpha) & =\sup \left\{x \in R \mid \mu_{A}(x) \geqslant \alpha\right\} \\
& =\left(a_{3}-a_{4}\right) \alpha+a_{4}, \\
A_{\text {Low }}^{-}(\alpha) & =\inf \left\{x \in R \mid v_{A}(x) \leqslant 1-\alpha\right\} \\
& =\left(b_{1}-b_{2}\right) \alpha+b_{2}, \\
A_{U p}^{-}(\alpha) & =\sup \left\{x \in R \mid v_{A}(x) \leqslant 1-\alpha\right\} \\
& =\left(b_{4}-b_{3}\right) \alpha+b_{3},
\end{aligned}
$$

ITFN are converted into interval valued intuitionistic fuzzy sets (IVIFS) e.g. (Ref. 24). $A=$ $\left\langle x,\left[A_{\text {Low }}^{+}(\alpha), A_{U p}^{+}(\alpha)\right],\left[A_{\text {Low }}^{-}(\alpha), A_{U p}^{-}(\alpha)\right]\right\rangle$.

\subsection{The belief rule-base structure}

In a "traditional" IF-THEN rule, decision makers state that the degree of an attribute affecting a consequent is either $100 \%$ true or $100 \%$ false e.g. (Ref. 2).To take into account belief degrees, attribute weights, and rule weights, the "traditional" rule is extended as e.g. (Ref. 11),

$$
\begin{aligned}
& R_{k}: \text { If }\left(X_{1}, \varepsilon_{1}\right) \text { is } A_{1}^{k} \wedge\left(X_{2}, \varepsilon_{2}\right) \text { is } A_{2}^{k} \wedge \cdots \wedge\left(X_{T_{k}},\right. \\
& \left.\quad \varepsilon_{T_{k}}\right) \text { is } A_{T_{k}}^{k}, \text { then }\left\{\left(D_{1}, \bar{\beta}_{1 k}\right),\left(D_{2}, \bar{\beta}_{2 k}\right), \cdots,\right. \\
& \left.\left(D_{N}, \bar{\beta}_{N k}\right)\right\} \text {, with a rule weight } \theta_{k} \text { and } \\
& \text { attribute weights } \delta_{k 1}, \delta_{k 2}, \cdots, \delta_{k T_{k}}, \\
& \text { and with } \bar{\beta}_{n k}(n \in\{1, \cdots, N\}),\left(\sum_{n=1}^{N} \bar{\beta}_{n k} \leqslant 1\right) .
\end{aligned}
$$

where $X_{i} \in\left\{A_{i}^{*}, i=1, \cdots, T_{k}\right\}, A_{i}^{*}$ is the input set, with the belief degree $\varepsilon_{i}$ to which the input is assessed to the corresponding evaluation grade, and $\varepsilon_{i} \geqslant 0, \Sigma_{i=1}^{T_{k}} \varepsilon_{i} \leqslant 1$. Two kinds of weight are considered. Those are the rule weight $\theta_{k}$ and attribute weights $\delta_{k i}$, with $\theta_{k} \geqslant 0, \sum_{k=1}^{L} \theta_{k}=1$ and in the $k$ th rule $\delta_{k i} \geqslant, \sum_{i=1}^{T_{k}} \delta_{k i}=1$. Given an input, if the rule weight $\theta_{k}$ is greater than zero, the corresponding rule will be activated.

The set $A_{i}^{k} \in\left\{A_{i j}, i=1, \cdots, T_{k}, j=\right.$ $\left.1, \cdots, J_{i}\right\}$ forms a collection of the referential values of antecedent attributes, where $T_{k}$ is the number of the antecedent attributes. $J_{i}$ is the number of the referential values of $i$ th antecedent attributes. Each referential value of antecedent attributes can be treated as an evaluation grade. The input set $A_{i}^{*}$ is assessed to the evaluation grade $A_{i}^{k}$ with belief degree $\alpha_{i j}$ The input assessment $S$ can be written as follows

$$
\begin{gathered}
S\left(A_{i}^{*}\right)=\left\{\left(A_{i j}, \alpha_{i j}\right), i=1, \cdots, T_{k}, j=1, \cdots, J_{i}\right\} . \\
D_{n}(n=1, \cdots, N) \text { is a set of consequents. } \bar{\beta}_{n k} \text { is }
\end{gathered}
$$
the degree to which $D_{n}$ is believed to be the consequent in the $k$ th rule. The belief degree $\bar{\beta}_{n k}$ can be assigned directly based on decision makers' experience, or by using some experimental data. The consequent assessment $S$ can be expressed as follows

$$
S\left(A_{i j}\right)=\left\{\left(D_{n}, \beta_{n k}\right), n=1, \cdots, N, k=1, \cdots, L\right\} .
$$

with $\beta_{n k} \geqslant 0, \sum_{n=1}^{N} \beta_{n k} \leqslant 1$. From inputs to consequents, information is transformed by belief degrees. Belief transfers from input $\varepsilon_{i}$ to updated consequent $\beta_{n k}$, through the intermediate links $\alpha_{i j}$ and $\bar{\beta}_{n k}$. Among these, $\varepsilon_{i}$ and $\bar{\beta}_{n k}$ is given by domain experts before making decision. One important task is to calculate the belief degree $\alpha_{i j}$.

The two sets $A_{i}^{*}$ and $A_{i}^{k}$ can be numeric or nonnumeric, continuous or discrete. Non-numeric attributes can be labeled by real numbers, traditional fuzzy sets, and generalized fuzzy sets. How to match the degree between two sets is an important step in the process of reasoning. Similarity measure, the $t$-norm function and $t$-conorm e.g. (Ref. 25), the area method for fuzzy sets e.g. (Ref. 26), and maxmin operation between fuzzy sets (e.g. (Ref. 11) and e.g. (Ref. 16))are used to compute the matching degree as shown below

$$
v\left(A_{i}^{*}, A_{i j}\right)=\max _{x}\left[\min \left(A_{i}^{*}(x), A_{i j}(x)\right)\right]
$$

However the max-min operation only considered extreme value. Grzegorzewski e.g. (Ref. 27) presented possible and necessary inclusion of IFSs.

\section{Intuitionistic fuzzy evidential reasoning}

In this section, an intuitionistic fuzzy evidential reasoning (IFER) method is proposed to perform reasoning. The flowchart of IFER is shown in Fig.1. 


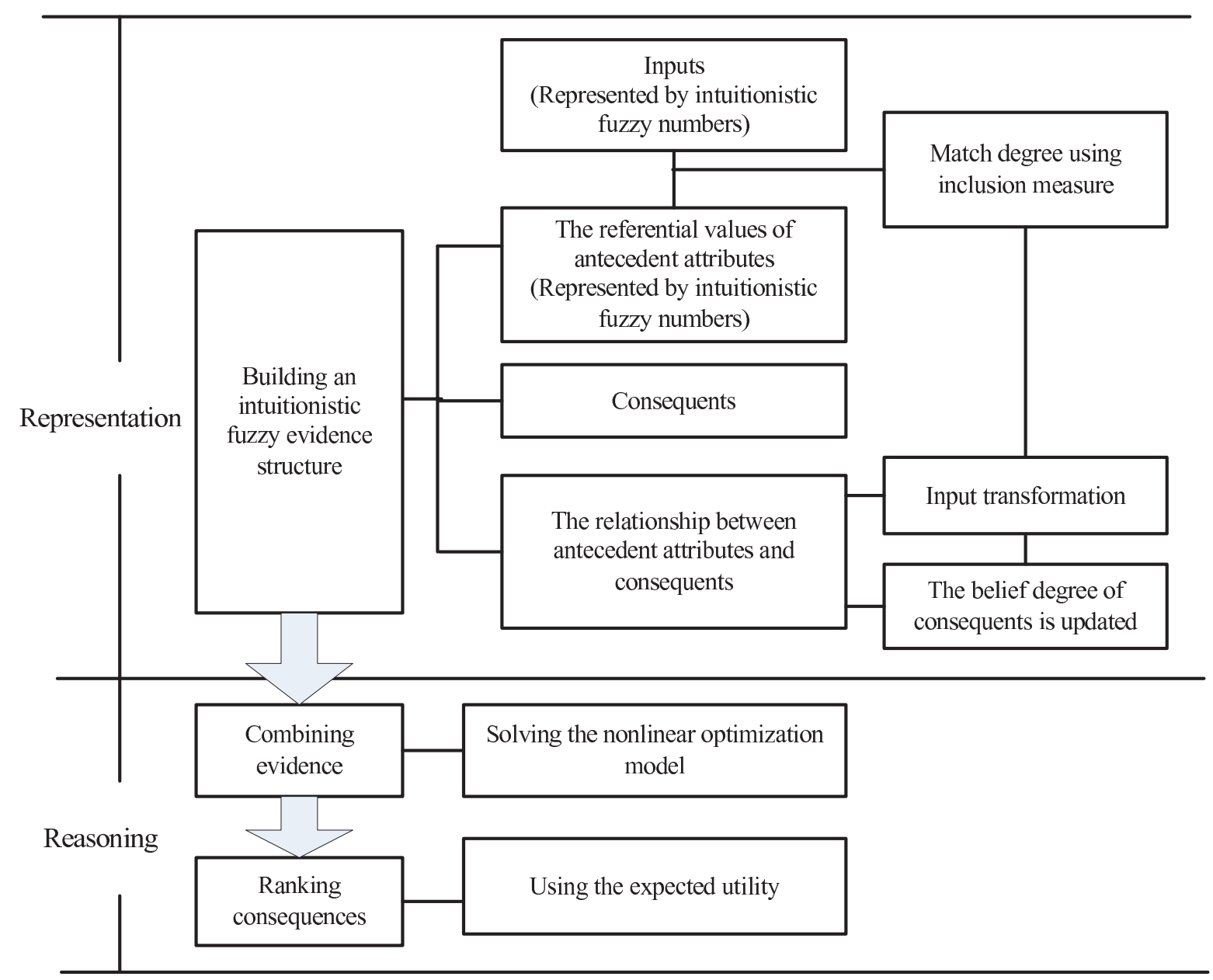

Fig. 1. The flowchart of intuitionistic fuzzy evidential reasoning (IFER).

\subsection{Intuitionistic fuzzy structure}

\subsubsection{The framework of evidence structure}

In evidential reasoning, the result depends on the suitable modeling of the domain knowledge and uncertainties. Dymova (e.g. (Ref. 18) and e.g. (Ref. 19)) proposed three hypotheses as Yes $(x \in A), N o(x \notin A)$ and (Yes,No)(hesitasion). $m(A)(Y e s), m(A)(N o), m(A)(Y e s, N o)$ represent the focal element of the basic assignment function respectively. The sum of them is equal to 1 .

Let $\Phi=\left\{\phi_{1}, \phi_{2}, \cdots, \phi_{T}\right\}$ be a set of collectively exhaustive and mutually exclusive hypotheses. It is called the frame of discernment. The $T$ assessment grades for all attributes, the referential values of an- tecedent attributes $A_{i j}$ is any subset of $\Phi$. The intuitionistic fuzzy structure is expressed as

$$
\begin{aligned}
\Re= & \left\{\left(A_{i j},\left[m\left(A_{i j}\right)(\text { Yes }), m\left(A_{i j}\right)(\text { Yes })+m\left(A_{i j}\right)(\text { Yes },\right.\right.\right. \\
& \left.\left.N o)], \mu_{A_{i j}}(x), v_{A_{i j}}(x)\right) \mid A_{i j} \in \Phi, x \in U\right\} \\
& \text { with } \quad i=1, \cdots, T_{k}, j=1, \cdots, J_{i} .
\end{aligned}
$$

where $A_{i j}$ denotes the fuzzy propositions in the frame of discernment $\Phi$. Among these, $m\left(A_{i j}\right)(Y e s)$ indicates the membership of belief mass assigned to the set $A_{i j} . \mathrm{U}$ represents the universe of discourse.

The belief interval $B I_{A}$ is a possibility interval $B I_{A}=\left[B e l_{A}, P l_{A}\right]$.The belief and plausibility of the 
intuitionistic fuzzy hypothesis $A$ are defined as

$$
\begin{gathered}
B e l_{A}=\sum_{j=1}^{J_{i}} \sum_{i=1}^{T_{k}}\left[m\left(A_{i j}\right)(Y e s)\right] \\
P l_{A}=\sum_{j=1}^{J_{i}} \sum_{i=1}^{T_{k}}\left[1-m\left(A_{i j}\right)(N o)\right]
\end{gathered}
$$

\subsubsection{Matching degree}

On the role of the input assessment $A_{i}^{*}$, the intuitionistic fuzzy belief degrees of antecedent attributes $A_{i j}$ are defined based on the following forms

$$
\begin{aligned}
\alpha_{i j_{\mu}}= & v\left(\mu_{A_{i}^{*}}(x), \mu_{A_{i j}}(x)\right) \varepsilon_{i}\left[\frac { 1 } { 2 } \left(\sum_{j=1}^{J_{i}} v\left(\mu_{A_{i}^{*}}(x), \mu_{A_{i j}}(x)\right)\right.\right. \\
& \left.\left.+\sum_{j=1}^{J_{i}} v\left(1-v_{A_{i}^{*}}(x), 1-v_{A_{i j}}(x)\right)\right)\right]^{-1} \\
\alpha_{i j_{v}}= & v\left(1-v_{A_{i}^{*}}(x), 1-v_{A_{i j}}(x)\right) \varepsilon_{i}\left[\frac { 1 } { 2 } \left(\sum _ { j = 1 } ^ { J _ { i } } v \left(\mu_{A_{i}^{*}}(x),\right.\right.\right. \\
& \left.\left.\left.\mu_{A_{i j}}(x)\right)+\sum_{j=1}^{J_{i}} v\left(1-v_{A_{i}^{*}}(x), 1-v_{A_{i j}}(x)\right)\right)\right]^{-1}
\end{aligned}
$$

$\mu_{A_{i}^{*}}(x), \quad v_{A_{i}^{*}}(x)$ are memberships and nonmemberships of input assessment $A_{i}^{*}$. $\mu_{A_{i j}}(x)$, $v_{A_{i j}}(x)$ are memberships and non-memberships of antecedent assessment $A_{i j}$, both of two sets are represented by intuitionistic fuzzy sets. According to e.g. "Eq. (3)", the first attribute $x_{1}$ of $A_{i}^{*}$ is converted by $\alpha$-cuts into $\left\langle x_{1},\left[A_{x_{1} \text { Low }}^{+*}, A_{x_{1} U p}^{+*}\right],\left[A_{x_{1} L o w}^{-*}, A_{x_{1} U p}^{-*}\right]\right\rangle$, and similarly, the $n$th attribute $x_{n}$ is $\left\langle x_{n},\left[A_{x_{n} L o w}^{+*}, A_{x_{n} U p}^{+*}\right],\left[A_{x_{n} L o w}^{-*}, A_{x_{n} U p}^{-*}\right]\right\rangle$. The lower fuzzy sets $A_{i}^{*-}$ e.g. ( (Ref. 27) corresponding to the first attribute $x_{1}$ of $A_{i}^{*}$ is $\left\langle x_{1},\left[A_{x_{1} L o w}^{+*}, A_{x_{1} U p}^{+*}\right]\right\rangle$. The upper fuzzy sets $A_{i}^{*+}$ corresponding to the first attribute $x_{1}$ of $A_{i}^{*}$ is $\left\langle x_{1},\left[A_{x_{1} L o w}^{-*}, A_{x_{1} U p}^{-*}\right]\right\rangle$.

Among these, operations on interval-valued fuzzy sets are available in the literature e.g. (Ref. 24). For any $A_{1}, A_{2}, \in I F S(X)$, the Hamming distance is $d\left(A_{1}, A_{2}\right)=\frac{1}{2} \sum_{i=1}^{n}\left(\mid \mu_{A_{1}}\left(x_{i}\right)-\right.$
$\left.\mu_{A_{2}}\left(x_{i}\right)|+| v_{A_{1}}\left(x_{i}\right)-v_{A_{2}}\left(x_{i}\right) \mid\right)$. In defuzzification and interval approximation of fuzzy sets, the approximation operator should maintain expected interval invariance e.g. (Ref. 28). During the process of transforming ITFN to IVIFS, the approximation operator of ITFN also maintains the expected interval of IVIFS. The intuitionistic fuzzy matching function based on inclusion measure e.g. (Ref. 27) is given as follows

$$
\begin{array}{r}
v\left(\mu_{A_{i}^{*}}(x), \mu_{A_{i j}}(x)\right)= \\
1-\left[\frac{1}{2} \int_{0}^{1}\left|A_{x_{i} L o w}^{-*}-\inf \left(A_{x_{i} L o w}^{-*}, A_{x_{i} L o w}^{+}\right)\right|^{p} d x\right. \\
\left.+\int_{0}^{1}\left|A_{x_{i} U p}^{-*}-\inf \left(A_{x_{i} U p}^{-*}, A_{x_{i} U p}^{+}\right)\right|^{p} d x\right]^{\frac{1}{p}} \\
v\left(1-v_{A_{i}^{*}}(x), 1-v_{A_{i j}}(x)\right)= \\
1-\left[\frac{1}{2} \int_{0}^{1}\left|A_{x_{i} L o w}^{+*}-\inf \left(A_{x_{i} L o w}^{+*}, A_{x_{i} L o w}^{-}\right)\right|^{p} d x\right. \\
\left.\quad+\int_{0}^{1}\left|A_{x_{i} U p}^{+*}-\inf \left(A_{x_{i} U p}^{+*}, A_{x_{i} U p}^{-}\right)\right|^{p} d x\right]^{\frac{1}{p}}
\end{array}
$$

\subsubsection{Weight assignment}

As mentioned by Tesfamariam e.g. (Ref. 29), credibility factors can be assigned in the same way as weight assignment. Aminravan e.g. (Ref. 16) also treated credibility factors $(C F)$ as weights. In this study, the same method is being adopted. Attribute weights are represented by $C F_{k 1}, C F_{k 2}, \cdots, C F_{k T_{k}}$. Normalization function for attribute weights is calculated by e.g. (Ref. 11),

$$
\begin{aligned}
& \bar{\delta}_{k i}=\frac{C F_{k i}}{\max _{i} C F_{k i}}, \\
& \quad \text { with }\left\{k=1, \cdots, L, i=1, \cdots, T_{k}\right\} .
\end{aligned}
$$

Using the simple weighted multiplicative aggregation function, we then calculate,

$$
\begin{aligned}
& \alpha_{k}=\Pi_{i=1}^{T_{k}}\left(\alpha_{i j_{k}}\right)^{\bar{\delta}_{k i}}, \\
& \quad \text { with }\left\{k=1, \cdots, L, i=1, \cdots, T_{k}, j=1, \cdots, J_{i}\right\},
\end{aligned}
$$


where $\alpha_{k}$ represents aggregation belief degree of the $k$ th rule, using the " $\wedge$ " connective for all antecedents attribute.

$$
\omega_{k}=\frac{\theta_{k} \alpha_{k}}{\sum_{k=1}^{L} \theta_{k} \alpha_{k}}, \text { with }\{k=1, \cdots, L\}
$$

Here $\theta_{k}$ represents the weight of the $k$ th rule. $\omega_{k}>0$ means that the $k$ th rule is activated.

\subsubsection{Belief degree}

After setting the matching degree, the relationship between input attributes and the antecedent attributes is obtained. In a rule, $A_{1}^{k}, A_{2}^{k}, \cdots, A_{T_{k}}^{k}$, which attribute participates in updating belief degrees is determined by function $\tau(i, k)$.If $A_{i}^{k}$ is used to transfer belief degree in a rule $R_{k}$, the value of $\tau(i, k)$ is 1. In other situations, its value is equal to 0 .

$\beta_{i k_{\mu}}=\bar{\beta}_{i k} \frac{\sum_{i=1}^{T_{k}}\left(\tau(i, k) \sum_{j=1}^{J_{i}} \alpha_{i j_{\mu}}\right)}{\sum_{i=1}^{T_{k}}(\tau(i, k)}$, with $\{k=1, \cdots, L\}$.

$\beta_{i k_{v}}=\bar{\beta}_{i k} \frac{\sum_{i=1}^{T_{k}}\left(\tau(i, k) \sum_{j=1}^{J_{i}} \alpha_{i j_{v}}\right)}{\sum_{i=1}^{T_{k}}(\tau(i, k)}$, with $\{k=1, \cdots, L\}$.

In the consequent assessment of the belief rule e.g. "Eq. (4)", the belief degrees $\bar{\beta}_{1 k}, \bar{\beta}_{2 k}, \cdots, \bar{\beta}_{N k}$ are given by domain experts. Under the role of inputs, the assessment
$S\left(A_{i j}\right)=\left\{\left(D_{1}, \bar{\beta}_{1 k}\right),\left(D_{2}, \bar{\beta}_{2 k}\right), \cdots,\left(D_{N}, \bar{\beta}_{N k}\right)\right\}$ $\left(\sum_{i=1}^{N} \bar{\beta}_{i k} \leqslant 1\right)$ is updated to $S\left(A_{i}^{*}\right)=$ $\left\{\left(D_{1},\left[\beta_{1 k_{\mu}}, \beta_{1 k_{v}}\right]\right),\left(D_{2},\left[\beta_{2 k_{\mu}}, \beta_{2 k_{v}}\right]\right), \cdots,\left(D_{N},\left[\beta_{N k_{\mu}}\right.\right.\right.$, $\left.\left.\left.\beta_{N k_{v}}\right]\right)\right\}$.

\subsection{Combination method}

As mentioned above, the consequent assessment is updated to interval belief degree, including lower and upper parts. After obtaining the interval belief degrees, they need to be converted to interval basic probability masses (BPA) by considering the relative weights. The interval BPA are $m_{n, k}=\left[\omega_{k} \beta_{n k_{\mu}}, \omega_{k} \beta_{n k_{v}}\right]$. If the assessment $S\left(A_{i}^{*}\right)$ is incomplete, the incomplete is due to two aspects. One is caused by the incomplete weights. That is $\bar{m}_{D, k}=1-\omega_{k}$. The other is caused by incomplete belief degrees. That is $\widetilde{m}_{D, k}=\left[\omega_{k} \beta_{D, k_{\mu}}, \omega_{k} \beta_{D, k_{v}}\right]$. The incomplete belief degrees of membership is that $\beta_{D, k_{\mu}}=\max \left(0,1-\sum_{n=1}^{N} \beta_{n k_{v}}\right)$. The incomplete belief degrees of non-membership is that $\beta_{D, k_{v}}=$ $\max \left(0,1-\sum_{n=1}^{N} \beta_{n k_{\mu}}\right)$. Because of this, DS theory can deal with the incomplete information which is ignored in other cases.

The interval probability masses are combined into overall interval belief degrees by solving the following nonlinear optimization models e.g. (Ref. 14). However, there is an initial condition. We require that the denominator of normalization factor is nonzero, that is $\sum_{n=1}^{N} \Pi_{k=1}^{L}\left(m_{n, k}+\bar{m}_{D, k}+\widetilde{m}_{D, k}\right)-(N-$ 1) $\Pi_{k=1}^{L}\left(\bar{m}_{D, k}+\widetilde{m}_{D, k}\right) \neq 0$.

$$
\begin{aligned}
& \operatorname{Max} / \text { Min } \beta_{n}\left(A_{i}^{*}\right)=\frac{\Pi_{k=1}^{L}\left(m_{n, k}+\bar{m}_{D, k}+\widetilde{m}_{D, k}\right)-\Pi_{k=1}^{L}\left(\bar{m}_{D, k}+\widetilde{m}_{D, k}\right)}{\left[\sum_{n=1}^{N} \Pi_{k=1}^{L}\left(m_{n, k}+\bar{m}_{D, k}+\widetilde{m}_{D, k}\right)-(N-1) \Pi_{k=1}^{L}\left(\bar{m}_{D, k}+\widetilde{m}_{D, k}\right)\right]-\left[\Pi_{k=1}^{L}\left(\bar{m}_{D, k}\right]\right.} \\
& \operatorname{Max} / \text { Min } \quad \beta_{D}\left(A_{i}^{*}\right)=\frac{\Pi_{k=1}^{L}\left(\bar{m}_{D, k}+\widetilde{m}_{D, k}\right)-\Pi_{k=1}^{L}\left(\bar{m}_{D, k}\right)}{\left[\sum_{n=1}^{N} \Pi_{k=1}^{L}\left(m_{n, k}+\bar{m}_{D, k}+\widetilde{m}_{D, k}\right)-(N-1) \Pi_{k=1}^{L}\left(\bar{m}_{D, k}+\widetilde{m}_{D, k}\right)\right]-\left[\Pi_{k=1}^{L}\left(\bar{m}_{D, k}\right]\right.} .
\end{aligned}
$$

\subsection{Ranking the consequents using expected utility}

Subject to

$$
\begin{aligned}
& \omega_{k} \beta_{n k_{\mu}} \leqslant m_{n, k} \leqslant \omega_{k} \beta_{n k_{v}} \\
& \omega_{k} \beta_{D, k_{\mu}} \leqslant \widetilde{m}_{D, k} \leqslant \omega_{k} \beta_{D, k_{v}} .
\end{aligned}
$$

For the combined distribution assessments, the ER approach usually employs expected utilities to com- 
pare or rank them e.g. (Ref. 14).

$$
\begin{aligned}
u_{\max }\left(A_{i}^{*}\right)= & \sum_{n=1}^{N-1} \beta_{n}\left(A_{i}^{*}\right) u\left(D_{n}\right)+\left(\beta_{N}\left(A_{i}^{*}\right)+\beta_{D}\left(A_{i}^{*}\right)\right) \\
& u\left(D_{N}\right), \quad i=1, \cdots, T_{k}, n=1, \cdots, N . \\
u_{\text {min }}\left(A_{i}^{*}\right)= & \left(\beta_{1}\left(A_{i}^{*}\right)+\beta_{D}\left(A_{i}^{*}\right)\right) u\left(D_{1}\right)+\sum_{n=2}^{N} \beta_{n}\left(A_{i}^{*}\right) \\
& u\left(D_{n}\right), \quad i=1, \cdots, T_{k}, n=1, \cdots, N .
\end{aligned}
$$

$$
u_{\text {aver }}\left(A_{i}^{*}\right)=\frac{u_{\max }\left(A_{i}^{*}\right)+u_{\min }\left(A_{i}^{*}\right)}{2}, \quad i=1, \cdots, T_{k} .
$$

$u\left(D_{n}\right)$ is the grade utility of consequent assessment $D_{n}$. And $\beta_{n}\left(A_{i}^{*}\right)$ is the belief degree to which $D_{n}$ is the consequent assessment if the input actives the antecedent $A_{i j}$.If the consequent $D_{N}$ is assigned to the most preferred assessment grade, $u\left(S\left(A_{i}^{*}\right)\right)=\sum_{n=1}^{N} \beta_{n}\left(A_{i}^{*}\right) u\left(D_{n}\right)$ achieves its maximum. If the consequent $D_{1}$ is assigned the least preferred assessment grade, $u\left(S\left(A_{i}^{*}\right)\right)$ achieves its minimum. And the max-min utility is calculated using nonlinear optimization models. The constraint conditions are the same as e.g. "Eq. (22)". As shown in e.g. "Eq. (25)", the midpoint of expected utility interval is used to rank all consequents.

\section{A numerical case study}

\subsection{A medical case description}

In this section, a medical case study is conducted for medical diagnosis. There are a number of steps to perform this case study.

- Step1: Determine the matching degree between inputs and the referential values of the antecedents in a rule using intuitionistic trapezoidal fuzzy inclusion measure,

- Step2: According to the matching degree, determine the intuitionistic fuzzy belief degrees,

- Step3: The belief degrees of consequent assessment in the belief rule-base $R_{k}$ (e.g. "Eq. (4)" )are updated to the interval belief degrees,
- Step4: Convert the interval belief degrees into interval basic probability assignments (BPA) using certainty factor $(C F)$ as the relative weight,

- Step5: Combine the interval BPA into overall interval belief degrees by solving the nonlinear optimization model,

- Step6: Rank the consequent assessment by assigning utilities to the interval belief degrees. The result can be obtained by midpoint of expected utility interval.

Patients' information can be treated as inputs, and they are represented by intuitionisitc trapezoidal fuzzy numbers (ITFN). Using ITFN to quantify linguistic values, it is shown e.g. "Table 1.". The corresponding relation of linguistic values and ITFN is determined by decision maker's experience. There are nine grades. For example e.g. (Ref. 30), lower level is corresponding to relatively small fuzzy numbers. Higher level is corresponding to big fuzzy numbers. However, every ITFN is composed of eight real numbers. These eight numbers are determined by the principle introduced in section 2.1 $\left(b_{1} \leqslant a_{1} \leqslant b_{2} \leqslant a_{2} \leqslant a_{3} \leqslant b_{3} \leqslant a_{4} \leqslant b_{4}\right)$.

Table 1. Linguistic values e.g. (Ref. 30) (determined by decision maker's experience) of intuitionistic trapezoidal fuzzy numbers (ITFN) for linguistic terms.

\begin{tabular}{lc}
\hline Linguistic terms & intuitionisitc trapezoidal fuzzy numbers \\
\hline Absolutely high & $\langle(1.0,1.0,1.0,1.0),(1.0,1.0,1.0,1.0)\rangle$ \\
Very high & $\langle(0.7,0.8,0.9,1.0),(0.7,0.8,0.9,1.0)\rangle$ \\
High & $\langle(0.5,0.6,0.7,0.8),(0.4,0.6,0.7,0.9)\rangle$ \\
Fairly high & $\langle(0.4,0.5,0.6,0.7),(0.3,0.5,0.6,0.8)\rangle$ \\
Medium & $\langle(0.3,0.4,0.5,0.6),(0.2,0.4,0.5,0.7)\rangle$ \\
Fairly low & $\langle(0.2,0.3,0.4,0.5),(0.1,0.3,0.4,0.6)\rangle$ \\
Low & $\langle(0.1,0.2,0.3,0.4),(0.0,0.2,0.3,0.5)\rangle$ \\
Very low & $\langle(0.0,0.1,0.2,0.3),(0.0,0.1,0.2,0.3)\rangle$ \\
Absolutely low & $\langle(0.0,0.0,0.0,0.0),(0.0,0.0,0.0,0.0)\rangle$ \\
\hline
\end{tabular}

There is a knowledge base for stroke diagnosis from the Peking University Third Hospital. It is shown as e.g. "Table A.1." (Appendix A). There are three consequents of stoke patients e.g. (Ref. 31). They are flaccid paralysis period, spastic period, convalescence period. The number of rules is 52 . $R 1-R 24$ is about flaccid paralysis period. $R 25-$ 
$R 28$ is about spastic period. $R 29-R 52$ is about convalescence period.

There is a patient, Jack. His four features are assessed as Activity of Daily Living (ADL) (High, $10 \%$; Medium, 60\%; Low, 30\%), Spasm (Fairly low, 10\%; Low, 85\%; Very low, 5\%), Muscle strength (Very high, 15\%; High, 75\%; Fairly High 10\%), Muscle tone (Fairly high, 20\%; Fairly low, 45\%; Low, 35\%). Linguistic values of four antecedent attributes are converted into intuitionistic trapezoidal fuzzy numbers.

The probable consequents 'belief degrees are given by decision maker. Before making decision, the information about consequents belief degree is evaluated according to decision maker's experience. It is also according to decision maker's subjective judgment, and it is as shown in e.g. "Table A.2." (Appendix A). Treating certainty factor as attribute weight. It is shown in e.g. "Table A.3." (Appendix A).

\subsection{Data training}

The intuitionistic fuzzy evidential reasoning algorithm is implemented as follows.

- Step1: Using e.g. "Eq. (13)" and "Eq. (14)" $(p=1)$ to calculate the matching degree $v\left(\mu_{A_{i}^{*}}(x), \mu_{A_{i j}}(x)\right), v\left(1-v_{A_{i}^{*}}(x), 1-v_{A_{i j}}(x)\right)$ between inputs assessment and antecedent assessment. Inclusion measure of the spastic period, $R 25-R 28$ is shown in e.g. "Table 2..".

Table 2. The inclusion measure $(\mathrm{p}=1)$ between inputs and antecedent assessment of the spastic period.

\begin{tabular}{ccccc}
\hline $\begin{array}{c}\text { Inclusion Measure } \\
\text { Spastic period }\end{array}$ & Activity of Daily Living & Spasm & Muscle strength & Muscle tone \\
\hline \multirow{4}{*}{$R$} & Medium & Low & High & Fairly Low \\
& {$[0.1453,0.1516]$} & {$[0.2147,0.2147]$} & {$[0.1816,0.1895]$} & {$[0.1137,0.1137]$} \\
& High & Fairly Low & Very High & Fairly High \\
& {$[0.0212,0.0265]$} & {$[0.0254,0.0265]$} & {$[0.0318,0.0397]$} & {$[0.0529,0.0529]$} \\
& Low & Very Low & Fairly High & Low \\
& {$[0.0750,0.0750]$} & {$[0.0125,0.0125]$} & {$[0.0250,0.0250]$} & {$[0.0875,0.0875]$} \\
& Medium & Low & High & Fairly Low \\
& {$[0.1453,0.1516]$} & {$[0.2147,0.2147]$} & {$[0.1816,0.1895]$} & {$[0.1137,0.1137]$} \\
$R 26$ & High & Fairly Low & Very High & Fairly High \\
& {$[0.0212,0.0265]$} & {$[0.0254,0.0265]$} & {$[0.0318,0.0397]$} & {$[0.0529,0.0529]$} \\
& Low & Very Low & Fairly High & Low \\
& {$[0.0750,0.0750]$} & {$[0.0125,0.0125]$} & {$[0.0250,0.0250]$} & {$[0.0875,0.0875]$} \\
& Medium & Low & High & Fairly Low \\
& {$[0.1237,0.1547]$} & {$[0.2191,0.2191]$} & {$[0.1853,0.1933]$} & {$[0.1160,0.1160]$} \\
$R 27$ & High & Fairly Low & Very High & Fairly High \\
& {$[0.0163,0.0272]$} & {$[0.0260,0.0272]$} & {$[0.0326,0.0408]$} & {$[0.0544,0.0544]$} \\
& Low & Very Low & Fairly High & Low \\
& {$[0.0723,0.0754]$} & {$[0.0126,0.0126]$} & {$[0.0251,0.0251]$} & {$[0.0880,0.0880]$} \\
& Medium & Low & High & Fairly Low \\
& {$[0.1277,0.1277]$} & {$[0.2262,0.2262]$} & {$[0.1912,0.1912]$} & {$[0.1197,0.1197]$} \\
& High & Fairly Low & Very High & Fairly High \\
& {$[0.0179,0.0179]$} & {$[0.0285,0.0285]$} & {$[0.0357,0.0357]$} & {$[0.0596,0.0596]$} \\
& Low & Very Low & Fairly High & Low \\
& {$[0.0726,0.0726]$} & {$[0.0126,0.0126]$} & {$[0.0253,0.0253]$} & {$[0.0884,0.0884]$} \\
\hline & & & &
\end{tabular}


- Step2: According to the matching degree, determine the intuitionistic fuzzy belief degrees $\alpha_{i j_{\mu}}$ and $\alpha_{i j_{v}}$ of antecedent attributes using e.g. "Eq. (11)" and "Eq. (12)".

- Step3: Using e.g. "Eq. (18)" and "Eq. (19)", the consequent assessment of the belief rulebase $R_{k}$ (e.g. "Eq. (4)" ) is updated to $\left\{\left(D_{1},\left[\beta_{1 k_{\mu}}, \beta_{1 k_{v}}\right]\right),\left(D_{2},\left[\beta_{2 k_{\mu}}, \beta_{2 k_{v}}\right]\right), \cdots,\left(D_{N},[\right.\right.$ $\left.\left.\left.\beta_{N k_{\mu}}, \beta_{N k_{v}}\right]\right)\right\}$. The updated belief degrees of consequent assessment $(R 1-R 24)$ are shown in e.g. "Table 3.”.

Table 3. The updated belief degree of consequent assessment.

\begin{tabular}{lrcc}
\hline Rule numbers & Flaccid paralysis period & Spastic period & Convalescence period \\
\hline$R 1$ & {$[0.1916,0.2564]$} & {$[0.0170,0.0228]$} & {$[0.0043,0.0057]$} \\
$R 2$ & {$[0.1947,0.2534]$} & {$[0.0151,0.0197]$} & {$[0.0065,0.0084]$} \\
$R 3$ & {$[0.1984,0.2448]$} & {$[0.0201,0.0248]$} & {$[0.0045,0.0055]$} \\
$R 4$ & {$[0.2104,0.2331]$} & {$[0.0189,0.0210]$} & {$[0.0071,0.0079]$} \\
$R 5$ & {$[0.2079,0.2308]$} & {$[0.0236,0.0262]$} & {$[0.0047,0.0052]$} \\
$R 6$ & {$[0.2105,0.2283]$} & {$[0.0215,0.0234]$} & {$[0.0072,0.0078]$} \\
$R 7$ & {$[0.2030,0.2311]$} & {$[0.0257,0.0292]$} & {$[0.0047,0.0053]$} \\
$R 8$ & {$[0.1881,0.2457]$} & {$[0.0216,0.0282]$} & {$[0.0065,0.0085]$} \\
$R 9$ & {$[0.1911,0.2378]$} & {$[0.0267,0.0332]$} & {$[0.0044,0.0055]$} \\
$R 10$ & {$[0.1970,0.2319]$} & {$[0.0252,0.0297]$} & {$[0.0069,0.0081]$} \\
$R 11$ & {$[0.1953,0.2287]$} & {$[0.0299,0.0350]$} & {$[0.0046,0.0054]$} \\
$R 12$ & {$[0.1978,0.2262]$} & {$[0.0279,0.0319]$} & {$[0.0070,0.0080]$} \\
$R 13$ & {$[0.1934,0.2252]$} & {$[0.0322,0.0375]$} & {$[0.0046,0.0054]$} \\
$R 14$ & {$[0.1959,0.2227]$} & {$[0.0303,0.0345]$} & {$[0.0070,0.0080]$} \\
$R 15$ & {$[0.1937,0.2200]$} & {$[0.0350,0.0398]$} & {$[0.0047,0.0053]$} \\
$R 16$ & {$[0.1961,0.2176]$} & {$[0.0331,0.0367]$} & {$[0.0071,0.0079]$} \\
$R 17$ & {$[0.1937,0.2153]$} & {$[0.0378,0.0420]$} & {$[0.0047,0.0053]$} \\
$R 18$ & {$[0.1960,0.2129]$} & {$[0.0359,0.0390]$} & {$[0.0072,0.0078]$} \\
$R 19$ & {$[0.1913,0.2130]$} & {$[0.0401,0.0447]$} & {$[0.0047,0.0053]$} \\
$R 20$ & {$[0.1889,0.2153]$} & {$[0.0373,0.0425]$} & {$[0.0070,0.0080]$} \\
$R 21$ & {$[0.1890,0.2103]$} & {$[0.0425,0.0473]$} & {$[0.0047,0.0053]$} \\
$R 22$ & {$[0.1913,0.2080]$} & {$[0.0407,0.0442]$} & {$[0.0072,0.0078]$} \\
$R 23$ & {$[0.1888,0.2057]$} & {$[0.0454,0.0495]$} & {$[0.0048,0.0052]$} \\
$R 24$ & {$[0.1969,0.1972]$} & {$[0.0449,0.0449]$} & {$[0.0075,0.0075]$} \\
\hline
\end{tabular}

- Step4: Normalization function $\bar{\delta}_{k i}$ for attribute weight is been calculated using e.g. "Eq. (15)". The rule weight $\omega_{k}$ is calculated using e.g. "Eq. (16)" and "Eq. (17)". Assign the relative weight to interval belief degrees. Interval basic probability assignments (BPA) are obtained.
- Step5: Combine the interval BPA into overall interval belief degrees $\beta_{n}\left(A_{i}^{*}\right)$ and $\beta_{N}\left(A_{i}^{*}\right)$ by solving the nonlinear optimization model using e.g. "Eq. (20)" and "Eq. (21)". These results are shown in e.g. "Table 4.".

Table 4. Combined overall interval belief degrees. 


\begin{tabular}{lccc}
\hline Classes & Flaccid paralysis period & Spastic period & Convalescence period \\
\hline$R 1-R 24$ & {$[0.2244,0.2605]$} & {$[0.3113,0.3793]$} & {$[0.0068,0.0084]$} \\
$R 25-R 28$ & {$[0.0136,0.0138]$} & {$[0.2213,0.2279]$} & {$[0.0191,0.0202]$} \\
$R 29-R 52$ & {$[0.0059,0.0076]$} & {$[0.0332,0.0372]$} & {$[0.2387,0.2621]$} \\
\hline
\end{tabular}

- Step6: Rank the consequent assessment by assigning utilities to the interval belief degrees $\beta_{1}\left(A_{i}^{*}\right), \beta_{n}\left(A_{i}^{*}\right), \beta_{N}\left(A_{i}^{*}\right)$ and $\beta_{D}\left(A_{i}^{*}\right)$ using e.g. "Eq. (23)", e.g. "Eq. (24)" and e.g. "Eq. (25)".
The result can be obtained by midpoint of expected utility interval $u_{\text {aver }}\left(A_{i}^{*}\right)$ in e.g. "Eq. (25)". From "Table 5.", patient Jack's feature is belonging to the convalescence period.

Table 5. The expected utilities of three periods and their ranking order.

\begin{tabular}{lccc}
\hline Expected utilities & Flaccid paralysis period & Spastic period & Convalescence period \\
\hline Minimum expected utility & 0.2135 & 0.2780 & 0.3534 \\
Maximum expected utility & 0.6550 & 0.7254 & 0.7865 \\
Average expected utility & 0.4343 & 0.5017 & 0.5701 \\
Rank & 3 & 2 & 1 \\
\hline
\end{tabular}

\subsection{Sensitivity analysis and comparative study}

\subsubsection{The sensitivity analysis of result with changed weights}

From e.g. "Fig. 2.", the weight of the attribute (Activity of Daily Living, ADL) changes with values $(0.0,0.1, \cdots, 1.0)$. Expected utilities of the flaccid paralysis period changed a lot. It is because of the attribute ADL is crucial for the flaccid paralysis pe- riod. The spastic period is not sensitive to the change of the weight of the attribute ADL. It is because of the attribute Spasm is crucial for the spastic period. However, from e.g. "Fig. 2.", we can see that the attribute ADL is also crucial for the convalescence period. It is because that in the convalescence period, the strokes patients' ability of ADL is also taken as an important evaluation for stroke patients' quality of life. 


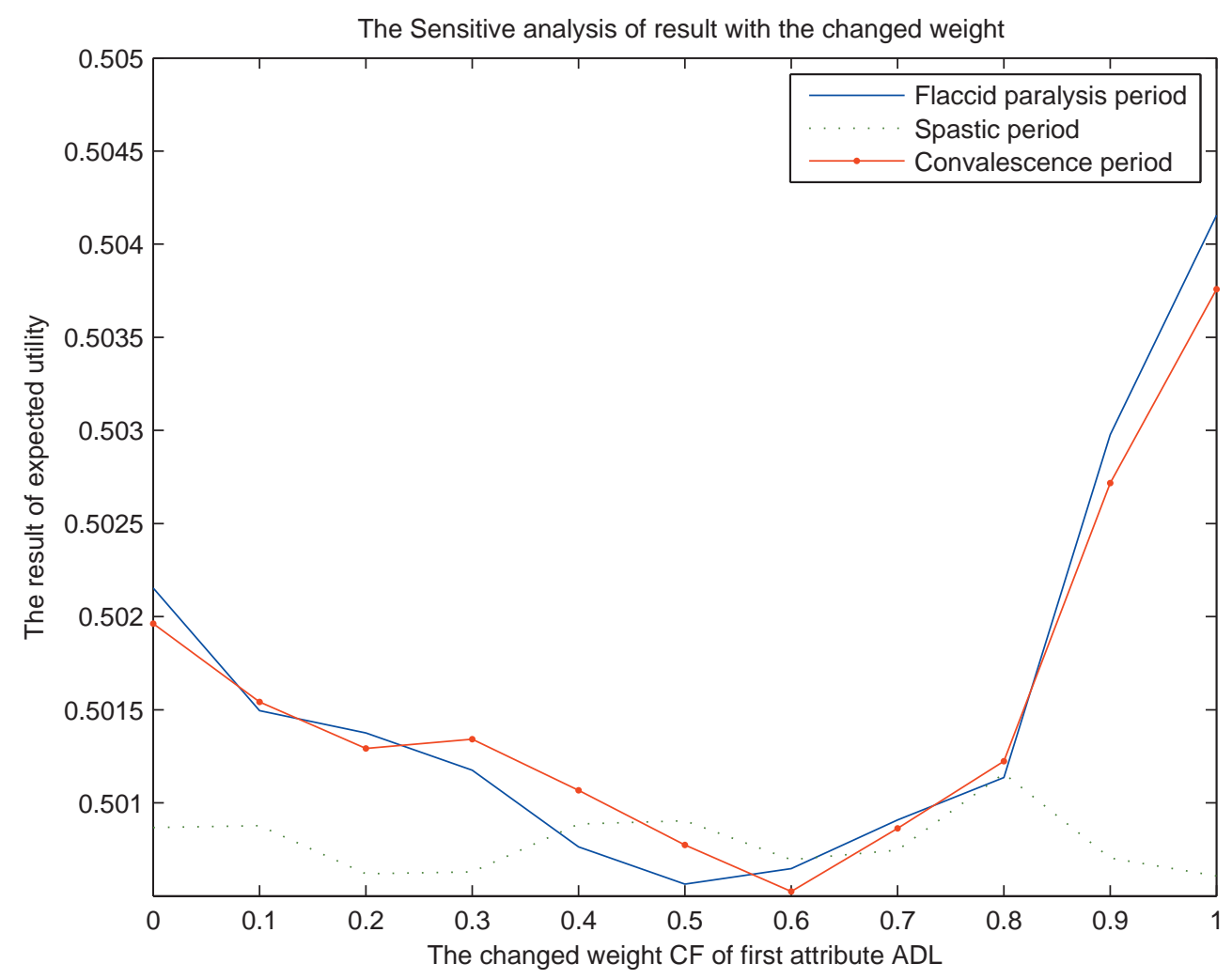

Fig. 2. The result of utilities with changed weight of first attribute-ADL.

\subsubsection{Comparative analysis}

Different from RIMER proposed in e.g. (Ref. 11), the inputs of this medical case are linguistic values. Linguistic inputs should be represented by intuitionistic trapezoidal fuzzy numbers in IFER. The match degrees are calculated between two fuzzy numbers. IGIB proposed in e.g. (Ref. 16) is used vague sets to represent evaluation grade. Inputs of IGIB case are real numbers. The match degrees are calculated between real numbers and fuzzy evaluation grade. Both RIMER and IGIB adopted max-min operation to set match degree. In Table 6, when match degree is calculated using max-min operation between intuitionistic trapezoidal fuzzy numbers, the rank of expected utilities is the same as the rank of IFER.

Table 6. The expected utilities of three periods and their ranking order using max-min operation.

\begin{tabular}{lccc}
\hline Expected utilities & Flaccid paralysis period & Spastic period & Convalescence period \\
\hline Minimum expected utility & 0.2060 & 0.2510 & 0.3538 \\
Maximum expected utility & 0.7201 & 0.7527 & 0.7865 \\
Average expected utility & 0.4631 & 0.5019 & 0.5702 \\
Rank & 3 & 2 & 1 \\
\hline
\end{tabular}




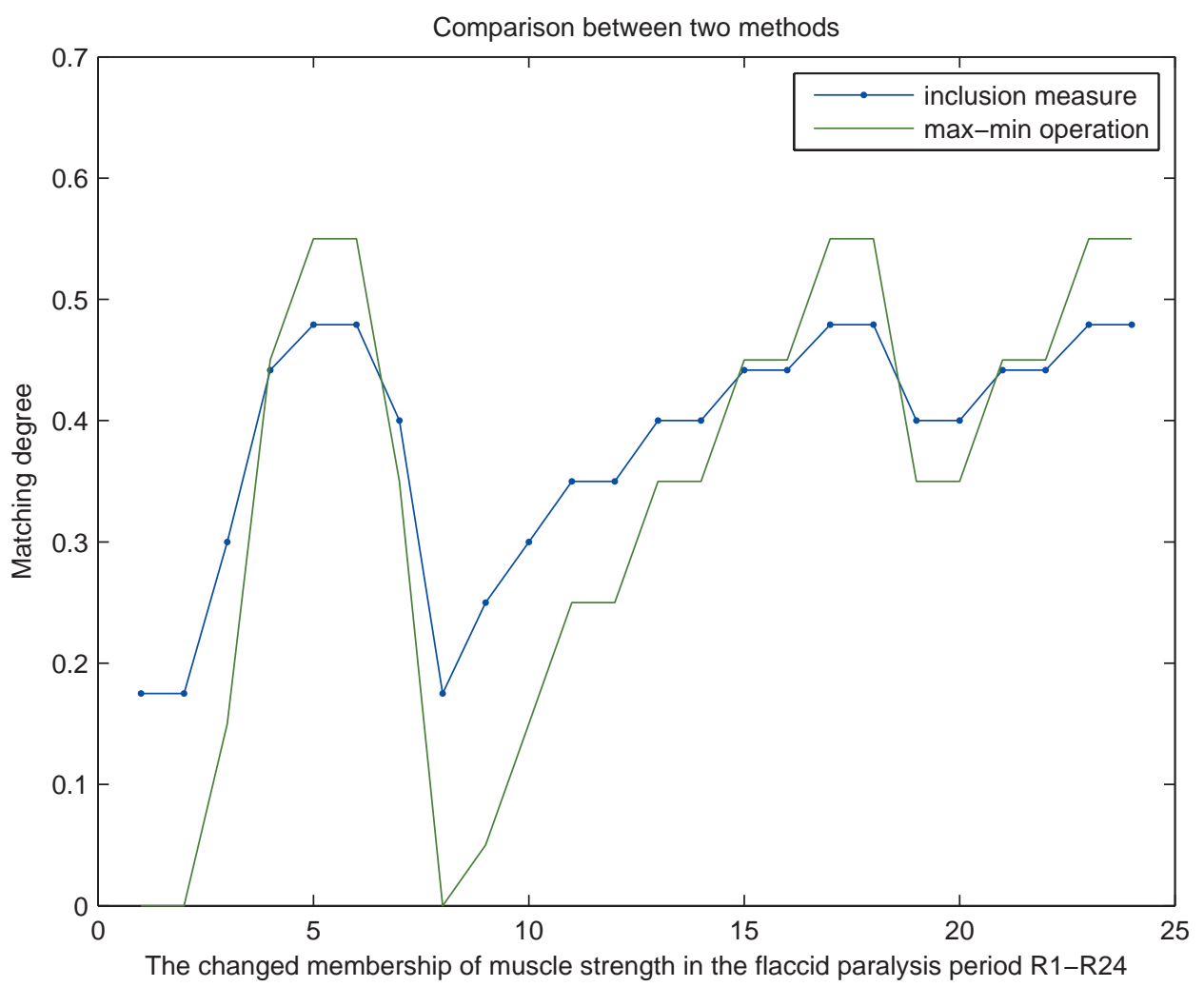

Fig. 3. The matching degree comparison between two methods (inclusion measure and max-min operation) with the changed membership of the third attribute (muscle strength) in the flaccid paralysis period.

From e.g. "Fig. 3.,we can see that, the matching degree using max-min operation obtains the extreme value 0 , when the membership of attribute is the extreme point $R 2$ and $R 8$. In the the point $R 5$ and $R 6$, the matching degree achieves the peak. The size of the peak using max-min operation is higher than the one using inclusion measure. Furthermore, the fluctuation range of curve using max-min operation is higher than the one using the inclusion measure. It can be concluded that the extreme value exhibits a more significant impact on the obtained matching degree using max-min operation. In some cases, this may lead to inaccurate results.

\section{Conclusions and further study}

The intuitionistic fuzzy evidential reasoning (IFER) arises as an interesting extension of the traditional fuzzy DS theory. IFER is a special case of RIMER with linguistic inputs. Based on the RIMER, different matching degree methods are adopted in terms of the type of inputs in IFER. Linguistic values are represented by intuitionistic trapezoidal fuzzy numbers. Inclusion measure is used to determine the matching degrees between inputs and antecedent attributes. Under the action of an input, if the rule weight is greater than zero, the corresponding rule becomes activated. For an activated rule, the belief degrees of the consequent assessment are updated according to the matching degrees. The reasoning model is mainly dependent on two parameters, at- 
tribute weight and belief degrees. Essentially, the key step of IFER is to obtain these two types of parameters. Through sensitivity analysis, the result of IFER is changed with parametric variation. Whether the decision making systems can produce accurate result mainly depends on the optimal learning of the parameters. In the future studies, the optimization of these parameters will be investigated in more detail.

\section{Acknowledgments}

Thank you for supporting by Program for Changjiang Scholars and Innovative Research Team in University under Grant IRT1208. Thank Professor Jian-bo Yang for providing guide on the RIMER methodology. Thank Professor Jie Lu and Professor Witold Pedrycz for revisions and comments, which further improve the quality of the paper.

\section{Appendix A}

Table A.1. Three periods of stroke pathological knowledge base represented using ITFN(Rule $1-$ Rule 24 for Flaccid paralysis period, Rule $25-$ Rule 28 for Spastic period, Rule $29-$ Rule 52 for Convalescence period ).

\begin{tabular}{|c|c|c|c|c|}
\hline $\begin{array}{l}\text { Rule numbers } \\
\text { Three periods }\end{array}$ & Activity of Daily Living & Spasm & Muscle strength & Muscle tone \\
\hline & Very high & Very low & Absolutely low & Very low \\
\hline Rule 1 & $\begin{array}{c}\langle(0.7,0.8,0.9,1.0), \\
(0.7,0.8,0.9,1.0)\rangle\end{array}$ & $\begin{array}{c}\langle(0.0,0.1,0.2,0.3), \\
(0.0,0.1,0.2,0.3)\rangle\end{array}$ & $\begin{array}{c}\langle(0.0,0.0,0.0,0.0), \\
(0.0,0.0,0.0,0.0)\rangle\end{array}$ & $\begin{array}{c}\langle(0.0,0.1,0.2,0.3), \\
(0.0,0.1,0.2,0.3)\rangle\end{array}$ \\
\hline & Very high & Very low & Absolutely low & Low \\
\hline Rule2 & $\begin{array}{c}\langle(0.7,0.8,0.9,1.0), \\
(0.7,0.8,0.9,1.0)\rangle\end{array}$ & $\begin{array}{c}\langle(0.0,0.1,0.2,0.3), \\
(0.0,0.1,0.2,0.3)\rangle\end{array}$ & $\begin{array}{c}\langle(0.0,0.0,0.0,0.0), \\
(0.0,0.0,0.0,0.0)\rangle\end{array}$ & $\begin{array}{c}\langle(0.1,0.2,0.3,0.4), \\
(0.0,0.2,0.3,0.5)\rangle\end{array}$ \\
\hline & Very high & Very low & Low & Very low \\
\hline Rule3 & $\begin{array}{c}\langle(0.7,0.8,0.9,1.0), \\
(0.7,0.8,0.9,1.0)\rangle\end{array}$ & $\begin{array}{c}\langle(0.0,0.1,0.2,0.3), \\
(0.0,0.1,0.2,0.3)\rangle\end{array}$ & $\begin{array}{c}\langle(0.1,0.2,0.3,0.4), \\
(0.0,0.2,0.3,0.5)\rangle\end{array}$ & $\begin{array}{c}\langle(0.0,0.1,0.2,0.3), \\
(0.0,0.1,0.2,0.3)\rangle\end{array}$ \\
\hline & Very high & Very low & Fairly high & Low \\
\hline Rule4 & $\begin{array}{c}\langle(0.7,0.8,0.9,1.0), \\
(0.7,0.8,0.9,1.0)\rangle\end{array}$ & $\begin{array}{c}\langle(0.0,0.1,0.2,0.3), \\
(0.0,0.1,0.2,0.3)\rangle\end{array}$ & $\begin{array}{c}\langle(0.4,0.5,0.6,0.7), \\
(0.3,0.5,0.6,0.8)\rangle\end{array}$ & $\begin{array}{c}\langle(0.1,0.2,0.3,0.4), \\
(0.0,0.2,0.3,0.5)\rangle\end{array}$ \\
\hline Rule5 & $\begin{array}{c}\text { Very high } \\
\langle(0.7,0.8,0.9,1.0), \\
(0.7,0.8,0.9,1.0)\rangle\end{array}$ & $\begin{array}{c}\text { Very low } \\
\langle(0.0,0.1,0.2,0.3), \\
(0.0,0.1,0.2,0.3)\rangle\end{array}$ & $\begin{array}{c}\text { High } \\
\langle(0.5,0.6,0.7,0.8), \\
(0.4,0.6,0.7,0.9)\rangle\end{array}$ & $\begin{array}{c}\text { Very low } \\
\langle(0.0,0.1,0.2,0.3), \\
(0.0,0.1,0.2,0.3)\rangle\end{array}$ \\
\hline Rule6 & $\begin{array}{c}\text { Very high } \\
\langle(0.7,0.8,0.9,1.0), \\
(0.7,0.8,0.9,1.0)\rangle\end{array}$ & $\begin{array}{c}\text { Very low } \\
\langle(0.0,0.1,0.2,0.3), \\
(0.0,0.1,0.2,0.3)\rangle\end{array}$ & $\begin{array}{c}\text { High } \\
\langle(0.5,0.6,0.7,0.8), \\
(0.4,0.6,0.7,0.9)\rangle\end{array}$ & $\begin{array}{c}\text { Low } \\
\langle(0.1,0.2,0.3,0.4), \\
(0.0,0.2,0.3,0.5)\rangle\end{array}$ \\
\hline Rule 7 & $\begin{array}{c}\text { Very high } \\
\langle(0.7,0.8,0.9,1.0), \\
(0.7,0.8,0.9,1.0)\rangle\end{array}$ & $\begin{array}{c}\text { Low } \\
\langle(0.1,0.2,0.3,0.4), \\
(0.0,0.2,0.3,0.5)\rangle\end{array}$ & $\begin{array}{c}\text { Medium } \\
\langle(0.3,0.4,0.5,0.6), \\
(0.2,0.4,0.5,0.7)\rangle\end{array}$ & $\begin{array}{c}\text { Very low } \\
\langle(0.0,0.1,0.2,0.3), \\
(0.0,0.1,0.2,0.3)\rangle\end{array}$ \\
\hline Rule8 & $\begin{array}{c}\text { Very high } \\
\langle(0.7,0.8,0.9,1.0), \\
(0.7,0.8,0.9,1.0)\rangle\end{array}$ & $\begin{array}{c}\text { Low } \\
\langle(0.1,0.2,0.3,0.4), \\
(0.0,0.2,0.3,0.5)\rangle\end{array}$ & $\begin{array}{c}\text { Absolutely low } \\
\langle(0.0,0.0,0.0,0.0), \\
(0.0,0.0,0.0,0.0)\rangle\end{array}$ & $\begin{array}{c}\text { Very low } \\
\langle(0.0,0.1,0.2,0.3), \\
(0.0,0.1,0.2,0.3)\rangle\end{array}$ \\
\hline Rule9 & $\begin{array}{c}\text { Very high } \\
\langle(0.7,0.8,0.9,1.0), \\
(0.7,0.8,0.9,1.0)\rangle\end{array}$ & $\begin{array}{c}\text { Low } \\
\langle(0.1,0.2,0.3,0.4), \\
(0.0,0.2,0.3,0.5)\rangle\end{array}$ & $\begin{array}{c}\text { Very low } \\
\langle(0.0,0.1,0.2,0.3), \\
(0.0,0.1,0.2,0.3)\rangle\end{array}$ & $\begin{array}{c}\text { Very low } \\
\langle(0.0,0.1,0.2,0.3), \\
(0.0,0.1,0.2,0.3)\rangle\end{array}$ \\
\hline Rule 10 & $\begin{array}{c}\text { Very high } \\
\langle(0.7,0.8,0.9,1.0), \\
(0.7,0.8,0.9,1.0)\rangle\end{array}$ & $\begin{array}{c}\text { Low } \\
\langle(0.1,0.2,0.3,0.4), \\
(0.0,0.2,0.3,0.5)\rangle\end{array}$ & $\begin{array}{c}\text { Low } \\
\langle(0.1,0.2,0.3,0.4), \\
(0.0,0.2,0.3,0.5)\rangle\end{array}$ & $\begin{array}{c}\text { Low } \\
\langle(0.1,0.2,0.3,0.4), \\
(0.0,0.2,0.3,0.5)\rangle\end{array}$ \\
\hline
\end{tabular}




\begin{tabular}{|c|c|c|c|c|}
\hline & Very high & Low & Fairly low & Very low \\
\hline \multirow[t]{2}{*}{ Rule11 } & $\begin{array}{c}\langle(0.7,0.8,0.9,1.0), \\
(0.7,0.8,0.9,1.0)\rangle\end{array}$ & $\begin{array}{c}\langle(0.1,0.2,0.3,0.4), \\
(0.0,0.2,0.3,0.5)\rangle\end{array}$ & $\begin{array}{c}\langle(0.2,0.3,0.4,0.5), \\
(0.1,0.3,0.4,0.6)\rangle\end{array}$ & $\begin{array}{c}\langle(0.0,0.1,0.2,0.3), \\
(0.0,0.1,0.2,0.3)\rangle\end{array}$ \\
\hline & Very high & Low & Fairly low & Low \\
\hline \multirow[t]{3}{*}{ Rule12 } & $\langle(0.7,0.8,0.9,1.0)$, & $\langle(0.1,0.2,0.3,0.4)$, & $\langle(0.2,0.3,0.4,0.5)$, & $\langle(0.1,0.2,0.3,0.4)$, \\
\hline & $(0.7,0.8,0.9,1.0)\rangle$ & $(0.0,0.2,0.3,0.5)\rangle$ & $(0.1,0.3,0.4,0.6)\rangle$ & $(0.0,0.2,0.3,0.5)\rangle$ \\
\hline & High & Very low & Medium & Very low \\
\hline \multirow[t]{3}{*}{ Rule 13} & $\langle(0.5,0.6,0.7,0.8)$, & $\langle(0.0,0.1,0.2,0.3)$, & $\langle(0.3,0.4,0.5,0.6)$, & $\langle(0.0,0.1,0.2,0.3)$, \\
\hline & $(0.4,0.6,0.7,0.9)\rangle$ & $(0.0,0.1,0.2,0.3)\rangle$ & $(0.2,0.4,0.5,0.7)\rangle$ & $(0.0,0.1,0.2,0.3)\rangle$ \\
\hline & High & Very low & Medium & Low \\
\hline \multirow[t]{3}{*}{ Rule14 } & $\langle(0.5,0.6,0.7,0.8)$, & $\langle(0.0,0.1,0.2,0.3)$, & $\langle(0.3,0.4,0.5,0.6)$, & $\langle(0.1,0.2,0.3,0.4)$, \\
\hline & $(0.4,0.6,0.7,0.9)\rangle$ & $(0.0,0.1,0.2,0.3)\rangle$ & $(0.2,0.4,0.5,0.7)\rangle$ & $(0.0,0.2,0.3,0.5)\rangle$ \\
\hline & High & Very low & Fairly high & Very low \\
\hline \multirow[t]{3}{*}{ Rule 15} & $\langle(0.5,0.6,0.7,0.8)$, & $\langle(0.0,0.1,0.2,0.3)$, & $\langle(0.4,0.5,0.6,0.7)$, & $\langle(0.0,0.1,0.2,0.3)$, \\
\hline & $(0.4,0.6,0.7,0.9)\rangle$ & $(0.0,0.1,0.2,0.3)\rangle$ & $(0.3,0.5,0.6,0.8)\rangle$ & $(0.0,0.1,0.2,0.3)\rangle$ \\
\hline & High & Very low & Fairly high & Low \\
\hline \multirow[t]{3}{*}{ Rule16 } & $\langle(0.5,0.6,0.7,0.8)$, & $\langle(0.0,0.1,0.2,0.3)$, & $\langle(0.4,0.5,0.6,0.7)$, & $\langle(0.1,0.2,0.3,0.4)$, \\
\hline & $(0.4,0.6,0.7,0.9)\rangle$ & $(0.0,0.1,0.2,0.3)\rangle$ & $(0.3,0.5,0.6,0.8)\rangle$ & $(0.0,0.2,0.3,0.5)\rangle$ \\
\hline & High & Very low & High & Very low \\
\hline \multirow[t]{3}{*}{ Rule 17} & $\langle(0.5,0.6,0.7,0.8)$, & $\langle(0.0,0.1,0.2,0.3)$, & $\langle(0.5,0.6,0.7,0.8)$, & $\langle(0.0,0.1,0.2,0.3)$, \\
\hline & $(0.4,0.6,0.7,0.9)\rangle$ & $(0.0,0.1,0.2,0.3)\rangle$ & $(0.4,0.6,0.7,0.9)\rangle$ & $(0.0,0.1,0.2,0.3)\rangle$ \\
\hline & High & Very low & High & Low \\
\hline \multirow[t]{3}{*}{ Rule 18} & $\langle(0.5,0.6,0.7,0.8)$, & $\langle(0.0,0.1,0.2,0.3)$, & $\langle(0.5,0.6,0.7,0.8)$, & $\langle(0.1,0.2,0.3,0.4)$, \\
\hline & $(0.4,0.6,0.7,0.9)\rangle$ & $(0.0,0.1,0.2,0.3)\rangle$ & $(0.4,0.6,0.7,0.9)\rangle$ & $(0.0,0.2,0.3,0.5)\rangle$ \\
\hline & High & Low & Medium & Low \\
\hline \multirow[t]{3}{*}{ Rule19 } & $\langle(0.5,0.6,0.7,0.8)$, & $\langle(0.1,0.2,0.3,0.4)$, & $\langle(0.3,0.4,0.5,0.6)$, & $\langle(0.1,0.2,0.3,0.4)$, \\
\hline & $(0.4,0.6,0.7,0.9)\rangle$ & $(0.0,0.2,0.3,0.5)\rangle$ & $(0.2,0.4,0.5,0.7)\rangle$ & $(0.0,0.2,0.3,0.5)\rangle$ \\
\hline & High & Low & Medium & Very low \\
\hline \multirow[t]{3}{*}{ Rule20 } & $\langle(0.5,0.6,0.7,0.8)$, & $\langle(0.1,0.2,0.3,0.4)$, & $\langle(0.3,0.4,0.5,0.6)$, & $\langle(0.0,0.1,0.2,0.3)$, \\
\hline & $(0.4,0.6,0.7,0.9)\rangle$ & $(0.0,0.2,0.3,0.5)\rangle$ & $(0.2,0.4,0.5,0.7)\rangle$ & $(0.0,0.1,0.2,0.3)\rangle$ \\
\hline & High & Low & Fairly high & Very low \\
\hline \multirow[t]{3}{*}{ Rule21 } & $\langle(0.5,0.6,0.7,0.8)$, & $\langle(0.1,0.2,0.3,0.4)$, & $\langle(0.4,0.5,0.6,0.7)$, & $\langle(0.0,0.1,0.2,0.3)$, \\
\hline & $(0.4,0.6,0.7,0.9)\rangle$ & $(0.0,0.2,0.3,0.5)\rangle$ & $(0.3,0.5,0.6,0.8)\rangle$ & $(0.0,0.1,0.2,0.3)\rangle$ \\
\hline & High & Low & Fairly high & Low \\
\hline \multirow[t]{3}{*}{ Rule22 } & $\langle(0.5,0.6,0.7,0.8)$, & $\langle(0.1,0.2,0.3,0.4)$, & $\langle(0.4,0.5,0.6,0.7)$, & $\langle(0.1,0.2,0.3,0.4)$, \\
\hline & $(0.4,0.6,0.7,0.9)\rangle$ & $(0.0,0.2,0.3,0.5)\rangle$ & $(0.3,0.5,0.6,0.8)\rangle$ & $(0.0,0.2,0.3,0.5)\rangle$ \\
\hline & High & Low & High & Very low \\
\hline \multirow[t]{3}{*}{ Rule23 } & $\langle(0.5,0.6,0.7,0.8)$, & $\langle(0.1,0.2,0.3,0.4)$, & $\langle(0.5,0.6,0.7,0.8)$, & $\langle(0.0,0.1,0.2,0.3)$, \\
\hline & $(0.4,0.6,0.7,0.9)\rangle$ & $(0.0,0.2,0.3,0.5)\rangle$ & $(0.4,0.6,0.7,0.9)\rangle$ & $(0.0,0.1,0.2,0.3)\rangle$ \\
\hline & High & Low & High & Very low \\
\hline \multirow[t]{3}{*}{ Rule24 } & $\langle(0.5,0.6,0.7,0.8)$, & $\langle(0.1,0.2,0.3,0.4)$, & $\langle(0.5,0.6,0.7,0.8)$, & $\langle(0.0,0.1,0.2,0.3)$, \\
\hline & $(0.4,0.6,0.7,0.9)\rangle$ & $(0.0,0.2,0.3,0.5)\rangle$ & $(0.4,0.6,0.7,0.9)\rangle$ & $(0.0,0.1,0.2,0.3)\rangle$ \\
\hline & Medium & Fairly low & High & High \\
\hline \multirow[t]{3}{*}{ Rule25 } & $\langle(0.3,0.4,0.5,0.6)$, & $\langle(0.2,0.3,0.4,0.5)$, & $\langle(0.5,0.6,0.7,0.8)$, & $\langle(0.5,0.6,0.7,0.8)$, \\
\hline & $(0.2,0.4,0.5,0.7)\rangle$ & $(0.1,0.3,0.4,0.6)\rangle$ & $(0.4,0.6,0.7,0.9)\rangle$ & $(0.4,0.6,0.7,0.9)\rangle$ \\
\hline & Medium & Fairly low & High & Very high \\
\hline \multirow[t]{3}{*}{ Rule26 } & $\langle(0.3,0.4,0.5,0.6)$, & $\langle(0.2,0.3,0.4,0.5)$, & $\langle(0.5,0.6,0.7,0.8)$, & $\langle(0.7,0.8,0.9,1.0)$, \\
\hline & $(0.2,0.4,0.5,0.7)\rangle$ & $(0.1,0.3,0.4,0.6)\rangle$ & $(0.4,0.6,0.7,0.9)\rangle$ & $(0.7,0.8,0.9,1.0)\rangle$ \\
\hline & Low & Fairly low & High & High \\
\hline \multirow[t]{2}{*}{ Rule27 } & $\langle(0.1,0.2,0.3,0.4)$, & $\langle(0.2,0.3,0.4,0.5)$, & $\langle(0.5,0.6,0.7,0.8)$, & $\langle(0.5,0.6,0.7,0.8)$, \\
\hline & $(0.0,0.2,0.3,0.5)\rangle$ & $(0.1,0.3,0.4,0.6)\rangle$ & $(0.4,0.6,0.7,0.9)\rangle$ & $(0.4,0.6,0.7,0.9)\rangle$ \\
\hline
\end{tabular}


Rule28

Rule29

Rule30

Rule31

Rule32

Rule33

Rule34

Rule35

Rule36

Rule37

Rule38

Rule39

Rule40

Rule41

Rule42

Rule43

Rule44
Low

$\langle(0.1,0.2,0.3,0.4)$, $(0.0,0.2,0.3,0.5)\rangle$ Low

$\langle(0.1,0.2,0.3,0.4)$, $(0.0,0.2,0.3,0.5)\rangle$ Low

$\langle(0.1,0.2,0.3,0.4)$, $(0.0,0.2,0.3,0.5)\rangle$ Low

$\langle(0.1,0.2,0.3,0.4)$, $(0.0,0.2,0.3,0.5)\rangle$ Low

$\langle(0.1,0.2,0.3,0.4)$, $(0.0,0.2,0.3,0.5)\rangle$ Low

$\langle(0.1,0.2,0.3,0.4)$, $(0.0,0.2,0.3,0.5)\rangle$

$$
\text { Low }
$$

$\langle(0.1,0.2,0.3,0.4)$, $(0.0,0.2,0.3,0.5)\rangle$ Low

$\langle(0.1,0.2,0.3,0.4)$, $(0.0,0.2,0.3,0.5)\rangle$ Low

$\langle(0.1,0.2,0.3,0.4)$, $(0.0,0.2,0.3,0.5)\rangle$ Low

$\langle(0.1,0.2,0.3,0.4)$, $(0.0,0.2,0.3,0.5)\rangle$ Low

$\langle(0.1,0.2,0.3,0.4)$, $(0.0,0.2,0.3,0.5)\rangle$ Low

$\langle(0.1,0.2,0.3,0.4)$ $(0.0,0.2,0.3,0.5)\rangle$ Low

$\langle(0.1,0.2,0.3,0.4)$, $(0.0,0.2,0.3,0.5)\rangle$

Very low

$\langle(0.0,0.1,0.2,0.3)$, $(0.0,0.1,0.2,0.3)\rangle$ Very low

$\langle(0.0,0.1,0.2,0.3)$, $(0.0,0.1,0.2,0.3)\rangle$

Very low

$\langle(0.0,0.1,0.2,0.3)$, $(0.0,0.1,0.2,0.3)\rangle$ Very low

$\langle(0.0,0.1,0.2,0.3)$, $(0.0,0.1,0.2,0.3)\rangle$
Fairly low

$\langle(0.2,0.3,0.4,0.5)$,

$(0.1,0.3,0.4,0.6)\rangle$

High

$\langle(0.5,0.6,0.7,0.8)$,

$(0.4,0.6,0.7,0.9)\rangle$

High

$\langle(0.5,0.6,0.7,0.8)$, $(0.4,0.6,0.7,0.9)\rangle$

High

$\langle(0.5,0.6,0.7,0.8)$, $(0.4,0.6,0.7,0.9)\rangle$

High

$\langle(0.5,0.6,0.7,0.8)$, $(0.4,0.6,0.7,0.9)\rangle$

High

$\langle(0.5,0.6,0.7,0.8)$, $(0.4,0.6,0.7,0.9)\rangle$

High

$\langle(0.5,0.6,0.7,0.8)$,

$(0.4,0.6,0.7,0.9)\rangle$

Very high

$\langle(0.7,0.8,0.9,1.0)$,

$(0.7,0.8,0.9,1.0)\rangle$

Very high

$\langle(0.7,0.8,0.9,1.0)$,

$(0.7,0.8,0.9,1.0)\rangle$

Very high

$\langle(0.7,0.8,0.9,1.0)$,

$(0.7,0.8,0.9,1.0)\rangle$

Very high

$\langle(0.7,0.8,0.9,1.0)$,

$(0.7,0.8,0.9,1.0)\rangle$

Very high

$\langle(0.7,0.8,0.9,1.0)$,

$(0.7,0.8,0.9,1.0)\rangle$

Very high

$\langle(0.7,0.8,0.9,1.0)$,

$(0.7,0.8,0.9,1.0)\rangle$

High

$\langle(0.5,0.6,0.7,0.8)$,

$(0.4,0.6,0.7,0.9)\rangle$

High

$\langle(0.5,0.6,0.7,0.8)$,

$(0.4,0.6,0.7,0.9)\rangle$

High

$\langle(0.5,0.6,0.7,0.8)$,

$(0.4,0.6,0.7,0.9)\rangle$

High

$\langle(0.5,0.6,0.7,0.8)$,

$(0.4,0.6,0.7,0.9)\rangle$
High

$\langle(0.5,0.6,0.7,0.8)$,

$(0.4,0.6,0.7,0.9)\rangle$

Very high

$\langle(0.7,0.8,0.9,1.0)$,

$(0.7,0.8,0.9,1.0)\rangle$

Very high

$\langle(0.7,0.8,0.9,1.0)$,

$(0.7,0.8,0.9,1.0)\rangle$

Very high

$\langle(0.7,0.8,0.9,1.0)$,

$(0.7,0.8,0.9,1.0)\rangle$

Absolutely high

$\langle(1.0,1.0,1.0,1.0)$,

$(1.0,1.0,1.0,1.0)\rangle$

Absolutely high

$\langle(1.0,1.0,1.0,1.0)$,

$(1.0,1.0,1.0,1.0)\rangle$

Absolutely high

$\langle(1.0,1.0,1.0,1.0)$,

$(1.0,1.0,1.0,1.0)\rangle$

Very high

$\langle(0.7,0.8,0.9,1.0)$,

$(0.7,0.8,0.9,1.0)\rangle$ Very high

$\langle(0.7,0.8,0.9,1.0)$,

$(0.7,0.8,0.9,1.0)\rangle$ Very high

$\langle(0.7,0.8,0.9,1.0)$,

$(0.7,0.8,0.9,1.0)\rangle$

Absolutely high

$\langle(1.0,1.0,1.0,1.0)$,

$(1.0,1.0,1.0,1.0)\rangle$

Absolutely high

$\langle(1.0,1.0,1.0,1.0)$,

$(1.0,1.0,1.0,1.0)\rangle$

Absolutely high

$\langle(1.0,1.0,1.0,1.0)$,

$(1.0,1.0,1.0,1.0)\rangle$

Very high

$\langle(0.7,0.8,0.9,1.0)$,

$(0.7,0.8,0.9,1.0)\rangle$

Very high

$\langle(0.7,0.8,0.9,1.0)$,

$(0.7,0.8,0.9,1.0)\rangle$

Very high

$\langle(0.7,0.8,0.9,1.0)$,

$(0.7,0.8,0.9,1.0)\rangle$

Absolutely high

$\langle(1.0,1.0,1.0,1.0)$,

$(1.0,1.0,1.0,1.0)\rangle$
Very high

$\langle(0.7,0.8,0.9,1.0)$,

$(0.7,0.8,0.9,1.0)\rangle$

Very low

$\langle(0.0,0.1,0.2,0.3)$

$(0.0,0.1,0.2,0.3)\rangle$

Low

$\langle(0.1,0.2,0.3,0.4)$, $(0.0,0.2,0.3,0.5)\rangle$

Fairly low

$\langle(0.2,0.3,0.4,0.5)$,

$(0.1,0.3,0.4,0.6)\rangle$

Very low

$\langle(0.0,0.1,0.2,0.3)$, $(0.0,0.1,0.2,0.3)\rangle$ Low

$\langle(0.1,0.2,0.3,0.4)$, $(0.0,0.2,0.3,0.5)\rangle$

Fairly low

$\langle(0.2,0.3,0.4,0.5)$, $(0.1,0.3,0.4,0.6)\rangle$

Very low

$\langle(0.0,0.1,0.2,0.3)$,

$(0.0,0.1,0.2,0.3)\rangle$

Low

$\langle(0.1,0.2,0.3,0.4)$, $(0.0,0.2,0.3,0.5)\rangle$

Fairly low

$\langle(0.2,0.3,0.4,0.5)$,

$(0.1,0.3,0.4,0.6)\rangle$

Very low

$\langle(0.0,0.1,0.2,0.3)$,

$(0.0,0.1,0.2,0.3)\rangle$

Low

$\langle(0.1,0.2,0.3,0.4)$ $(0.0,0.2,0.3,0.5)\rangle$

Fairly low

$\langle(0.2,0.3,0.4,0.5)$,

$(0.1,0.3,0.4,0.6)\rangle$

Very low

$\langle(0.0,0.1,0.2,0.3)$, $(0.0,0.1,0.2,0.3)\rangle$ Low

$\langle(0.1,0.2,0.3,0.4)$, $(0.0,0.2,0.3,0.5)\rangle$

Fairly low

$\langle(0.2,0.3,0.4,0.5)$, $(0.1,0.3,0.4,0.6)\rangle$

Very low

$\langle(0.0,0.1,0.2,0.3)$, $(0.0,0.1,0.2,0.3)\rangle$ 


\begin{tabular}{ccccc} 
& Very low & High & Absolutely high & Low \\
Rule45 & $\langle(0.0,0.1,0.2,0.3)$, & $\langle(0.5,0.6,0.7,0.8)$, & $\langle(1.0,1.0,1.0,1.0)$, & $\langle(0.1,0.2,0.3,0.4)$, \\
& $(0.0,0.1,0.2,0.3)\rangle$ & $(0.4,0.6,0.7,0.9)\rangle$ & $(1.0,1.0,1.0,1.0)\rangle$ & $(0.0,0.2,0.3,0.5)\rangle$ \\
& Very low & High & Absolutely high & Fairly low \\
Rule46 & $\langle(0.0,0.1,0.2,0.3)$, & $\langle(0.5,0.6,0.7,0.8)$, & $\langle(1.0,1.0,1.0,1.0)$, & $\langle(0.2,0.3,0.4,0.5)$, \\
& $(0.0,0.1,0.2,0.3)\rangle$ & $(0.4,0.6,0.7,0.9)\rangle$ & $(1.0,1.0,1.0,1.0)\rangle$ & $(0.1,0.3,0.4,0.6)\rangle$ \\
& Very low & Very high & Very high & Very low \\
Rule47 & $\langle(0.0,0.1,0.2,0.3)$, & $\langle(0.7,0.8,0.9,1.0)$, & $\langle(0.7,0.8,0.9,1.0)$, & $\langle(0.0,0.1,0.2,0.3)$, \\
& $(0.0,0.1,0.2,0.3)\rangle$ & $(0.7,0.8,0.9,1.0)\rangle$ & $(0.7,0.8,0.9,1.0)\rangle$ & $(0.0,0.1,0.2,0.3)\rangle$ \\
& Very low & Very high & Very high & Low \\
Rule48 & $\langle(0.0,0.1,0.2,0.3)$, & $\langle(0.7,0.8,0.9,1.0)$, & $\langle(0.7,0.8,0.9,1.0)$, & $\langle(0.1,0.2,0.3,0.4)$, \\
& $(0.0,0.1,0.2,0.3)\rangle$ & $(0.7,0.8,0.9,1.0)\rangle$ & $(0.7,0.8,0.9,1.0)\rangle$ & $(0.0,0.2,0.3,0.5)\rangle$ \\
& Very low & Very high & Very high & Fairly low \\
Rule49 & $\langle(0.0,0.1,0.2,0.3)$, & $\langle(0.7,0.8,0.9,1.0)$, & $\langle(0.7,0.8,0.9,1.0)$, & $\langle(0.2,0.3,0.4,0.5)$, \\
& $(0.0,0.1,0.2,0.3)\rangle$ & $(0.7,0.8,0.9,1.0)\rangle$ & $(0.7,0.8,0.9,1.0)\rangle$ & $(0.1,0.3,0.4,0.6)\rangle$ \\
Rule50 & Very low & Very high & Absolutely high & Very low \\
& $\langle(0.0,0.1,0.2,0.3)$, & $\langle(0.7,0.8,0.9,1.0)$, & $\langle(1.0,1.0,1.0,1.0)$, & $\langle(0.0,0.1,0.2,0.3)$, \\
& $(0.0,0.1,0.2,0.3)\rangle$ & $(0.7,0.8,0.9,1.0)\rangle$ & $(1.0,1.0,1.0,1.0)\rangle$ & $(0.0,0.1,0.2,0.3)\rangle$ \\
Rule51 & Very low & Very high & Absolutely high & Low \\
& $\langle(0.0,0.1,0.2,0.3)$, & $\langle(0.7,0.8,0.9,1.0)$, & $\langle(1.0,1.0,1.0,1.0)$, & $\langle(0.1,0.2,0.3,0.4)$, \\
& $(0.0,0.1,0.2,0.3)\rangle$ & $(0.7,0.8,0.9,1.0)\rangle$ & $(1.0,1.0,1.0,1.0)\rangle$ & $(0.0,0.2,0.3,0.5)\rangle$ \\
Rule52 & Very low & Very high & Absolutely high & Fairly low \\
& $\langle(0.0,0.1,0.2,0.3)$, & $\langle(0.7,0.8,0.9,1.0)$, & $\langle(1.0,1.0,1.0,1.0)$, & $\langle(0.2,0.3,0.4,0.5)$, \\
& $(0.0,0.1,0.2,0.3)\rangle$ & $(0.7,0.8,0.9,1.0)\rangle$ & $(1.0,1.0,1.0,1.0)\rangle$ & $(0.1,0.3,0.4,0.6)\rangle$ \\
\hline
\end{tabular}

Table A.2. The initial belief degree of consequent assessment.

\begin{tabular}{lccc}
\hline Rule numbers & Flaccid paralysis period & Spastic period & Convalescence period \\
\hline Rule 1 & 0.90 & 0.08 & 0.02 \\
Rule 2 & 0.90 & 0.07 & 0.03 \\
Rule3 & 0.89 & 0.09 & 0.02 \\
Rule 4 & 0.89 & 0.08 & 0.03 \\
Rule5 & 0.88 & 0.10 & 0.02 \\
Rule6 & 0.88 & 0.09 & 0.03 \\
Rule7 & 0.87 & 0.11 & 0.02 \\
Rule8 & 0.87 & 0.10 & 0.03 \\
Rule 9 & 0.86 & 0.12 & 0.02 \\
Rule 10 & 0.86 & 0.11 & 0.03 \\
Rule 11 & 0.85 & 0.13 & 0.02 \\
Rule 12 & 0.85 & 0.12 & 0.03 \\
Rule 13 & 0.84 & 0.14 & 0.02 \\
Rule 14 & 0.84 & 0.13 & 0.03 \\
Rule 15 & 0.83 & 0.15 & 0.02 \\
Rule 16 & 0.83 & 0.14 & 0.03 \\
Rule 17 & 0.82 & 0.16 & 0.02 \\
Rule 18 & 0.82 & 0.15 & 0.03 \\
Rule 19 & 0.81 & 0.17 & 0.02 \\
Rule20 & 0.81 & 0.16 & 0.03 \\
Rule21 & 0.80 & 0.18 & 0.02 \\
Rule22 & 0.80 & 0.17 & 0.03
\end{tabular}




\begin{tabular}{llll} 
Rule 23 & 0.79 & 0.19 & 0.02 \\
Rule 24 & 0.79 & 0.18 & 0.03 \\
Rule25 & 0.10 & 0.80 & 0.10 \\
Rule26 & 0.09 & 0.85 & 0.06 \\
Rule27 & 0.05 & 0.88 & 0.07 \\
Rule28 & 0.02 & 0.90 & 0.08 \\
Rule29 & 0.02 & 0.19 & 0.79 \\
Rule30 & 0.03 & 0.18 & 0.79 \\
Rule31 & 0.02 & 0.17 & 0.80 \\
Rule32 & 0.03 & 0.17 & 0.80 \\
Rule33 & 0.02 & 0.16 & 0.81 \\
Rule34 & 0.03 & 0.16 & 0.81 \\
Rule35 & 0.02 & 0.15 & 0.82 \\
Rule36 & 0.15 & 0.82 \\
Rule37 37 & 0.83 \\
Rule38 & 0.03 & 0.14 & 0.83 \\
Rule39 & 0.02 & 0.14 & 0.84 \\
Rule40 & 0.03 & 0.13 & 0.84 \\
Rule41 & 0.13 & 0.85 \\
Rule42 & 0.02 & 0.12 & 0.85 \\
Rule43 & 0.03 & 0.12 & 0.86 \\
Rule44 & 0.02 & 0.11 & 0.86 \\
Rule45 & 0.03 & 0.11 & 0.87 \\
Rule46 & 0.10 & 0.87 \\
Rule47 & 0.10 & 0.88 \\
Rule48 & 0.09 & 0.88 \\
Rule49 & 0.09 & 0.89 \\
Rule50 & 0.03 & 0.08 & 0.90 \\
Rule51 & 0.07 & \\
Rule52 & 0.02 & & \\
\hline & 0.03 & 03 & 0.90 \\
\hline
\end{tabular}

Table A.3. The certainty factor of three periods.

\begin{tabular}{lcccc}
\hline Rule numbers & Activity of Daily Living & Spasm & Muscle strength & Muscle tone \\
\hline Rule 1 & 0.8 & 0.5 & 0.5 & 0.8 \\
Rule 2 & 0.8 & 0.5 & 0.5 & 1.0 \\
Rule 3 & 0.8 & 0.5 & 0.8 & 0.8 \\
Rule 4 & 0.8 & 0.5 & 0.8 & 1.0 \\
Rule5 & 0.8 & 0.5 & 1.0 & 0.8 \\
Rule6 & 0.8 & 0.5 & 1.0 & 1.0 \\
Rule 7 & 0.8 & 0.8 & 0.5 & 0.8 \\
Rule8 & 0.8 & 0.8 & 0.5 & 1.0 \\
Rule 9 & 0.8 & 0.8 & 0.8 & 0.8 \\
Rule 10 & 0.8 & 0.8 & 0.8 & 1.0 \\
Rule 11 & 0.8 & 0.8 & 1.0 & 0.8 \\
Rule 12 & 0.8 & 0.8 & 1.0 & 1.0 \\
Rule 13 & 0.8 & 0.5 & 0.5 & 0.8 \\
Rule 14 & 0.8 & 0.5 & 0.5 & 1.0 \\
Rule 15 & 0.8 & 0.5 & 0.8 & 0.8
\end{tabular}




\begin{tabular}{|c|c|c|c|c|}
\hline Rule16 & 0.8 & 0.5 & 0.8 & 1.0 \\
\hline Rule17 & 0.8 & 0.5 & 1.0 & 0.8 \\
\hline Rule18 & 0.8 & 0.5 & 1.0 & 1.0 \\
\hline Rule19 & 0.8 & 0.8 & 0.5 & 0.8 \\
\hline Rule20 & 0.8 & 0.8 & 0.5 & 1.0 \\
\hline Rule21 & 0.8 & 0.8 & 0.8 & 0.8 \\
\hline Rule22 & 0.8 & 0.8 & 0.8 & 1.0 \\
\hline Rule23 & 0.8 & 0.8 & 1.0 & 0.8 \\
\hline Rule24 & 0.8 & 0.8 & 1.0 & 1.0 \\
\hline Rule25 & 0.8 & 0.8 & 0.3 & 0.8 \\
\hline Rule26 & 0.8 & 0.8 & 0.3 & 0.5 \\
\hline Rule27 & 0.5 & 0.8 & 0.3 & 0.8 \\
\hline Rule28 & 0.5 & 0.8 & 0.3 & 0.5 \\
\hline Rule29 & 0.8 & 0.5 & 0.8 & 0.5 \\
\hline Rule30 & 0.8 & 0.5 & 0.8 & 0.8 \\
\hline Rule31 & 0.8 & 0.5 & 0.8 & 0.8 \\
\hline Rule32 & 0.8 & 0.5 & 0.5 & 0.5 \\
\hline Rule33 & 0.8 & 0.5 & 0.5 & 0.8 \\
\hline Rule34 & 0.8 & 0.5 & 0.5 & 0.8 \\
\hline Rule35 & 0.8 & 0.5 & 0.8 & 0.5 \\
\hline Rule36 & 0.8 & 0.5 & 0.8 & 0.8 \\
\hline Rule37 & 0.8 & 0.5 & 0.8 & 0.8 \\
\hline Rule38 & 0.8 & 0.5 & 0.5 & 0.5 \\
\hline Rule39 & 0.8 & 0.5 & 0.5 & 0.8 \\
\hline Rule40 & 0.8 & 0.5 & 0.5 & 0.8 \\
\hline Rule41 & 0.5 & 0.5 & 0.8 & 0.5 \\
\hline Rule42 & 0.5 & 0.5 & 0.8 & 0.8 \\
\hline Rule43 & 0.5 & 0.5 & 0.8 & 0.8 \\
\hline Rule44 & 0.5 & 0.5 & 0.5 & 0.5 \\
\hline Rule45 & 0.5 & 0.5 & 0.5 & 0.8 \\
\hline Rule46 & 0.5 & 0.5 & 0.5 & 0.8 \\
\hline Rule47 & 0.5 & 0.5 & 0.8 & 0.5 \\
\hline Rule 48 & 0.5 & 0.5 & 0.8 & 0.8 \\
\hline Rule49 & 0.5 & 0.5 & 0.8 & 0.8 \\
\hline Rule50 & 0.5 & 0.5 & 0.5 & 0.5 \\
\hline Rule51 & 0.5 & 0.5 & 0.5 & 0.8 \\
\hline Rule52 & 0.5 & 0.5 & 0.5 & 0.8 \\
\hline
\end{tabular}

\section{References}

1. T. J. Ross, "Fuzzy logic with engineering applications (2nd ed.)," New York: JohnWiley \& Sons, 2-12 (2004).

2. G. L. Kong, D. L.Xu, X.B. Liu and J.B. Yang "Applying a belief rule-base inference methodology to a guideline-based clinical decision support system," $E x$ pert Systems, 26(5), 391-408 (2009).

3. L. Lin, P.J.-H. Hu, and O.R. Liu Sheng, "A decision support system for lower back pain diagnosis uncertainty management and clinical evaluation," Decision Support System, 42(2) , 1152-1169(2006).

4. R. W. Jones, A. Lowe and M. J. Harrison, "A frame- work for intelligent medical diagnosis using the theory of evidence," Knowledge-Based Systems, 15, 7784 (2002).

5. I.N. Durbach and T. J. Stewart, "Modeling uncertainty in multi-criteria decision analysis," European Journal of Operational Research, 223, 1-14 (2012).

6. D. Xu, "An introduction and survey of the evidential reasoning approach for multiple criteria decision analysis," Annals of Operations Research, 195(1), 163187 (2012).

7. J. Yen, "Generalizing the Dempster-Shafer theory to fuzzy sets," IEEE Transactions on Systems, Man, and Cybernetics, 20(3), 559-569 (1990). 
8. E. Binaghi and P. Madella, "Fuzzy Dempster-Shafer reasoning for rule-based classifiers," International Journal of Intelligent Systems, 14(6), 559-583 (1999).

9. H. Zhu and O. Basir, "An adaptive fuzzy evidential nearest neighbor formulation for classifying remote sensing images," IEEE Transactions on Geoscience and Remote Sensing, 43(8), 1874-1889 (2005).

10. H. Zhu and O. Basir, "A novel fuzzy evidential reasoning paradigm for data fusion with applications in image processing," Soft Computer, 10(12), 1169-1180 (2006).

11. J.B. Yang, J. Liu, and J. Wang, "Belief rule-base inference methodology using the evidential reasoning approach-RIMER," IEEE transaction on systems, man, and cybernetics-part A: systems and humans, 36(2), 266-285 (2006).

12. P. Sevastianov , L. Dymova and P. Bartosiewicz, "A framework for rule-base evidential reasoning in the interval setting applied to diagnosing type 2 diabetes," Expert Systems with Applications, 39, 41904200 (2012).

13. V.N. Huynh, Y. Nakamori, T.B. Ho and T. Murai, "Multiple-Attribute Decision Making Under Uncertainty: The Evidential Reasoning Approach Revisited," IEEE transaction on systems, man, and cybernetics-part A: systems and humans, 36(4), 804822 (2006).

14. Y.M. Wang, J.B. Yang, D.L. Xu, and K.S. Chin, "The evidential reasoning approach for multiple attribute decision analysis using interval belief degrees," $E u$ ropean Journal of Operational Research, 175, 35-66 (2006).

15. M. Guo , J.B. Yang, K.S. Chin , and H.W. Wang, "Evidential reasoning based preference programming for multiple attribute decision analysis under uncertainty," European Journal of Operational Research, 182, 1294-1312 (2007).

16. R. Aminravan, M. Sadiq, M. Hoorfar, A. Rodriguez, H. Francisque and Najjaran, "Evidential reasoning using extended fuzzy Dempster-Shafer theory for handling various facets of information deficiency," International Journal of Intelligent Systems, 26, 731-758 (2011).

17. S. Husain, Y. Ahmad, and M. A. Alam, "A study on the Role of Intuitionistic Fuzzy Set in Decision making problems," International Journal of Computer Applications(0975 - 888), 48(19), 35-41 (2012).

18. L. Dymova and P. Sevastjanov, "An interpretation of intuitionistic fuzzy sets in term of evidence theory: de- cision making aspect," Knowledge-Based Systems, 23, 772-782 (2010).

19. L. Dymova and P. Sevastjanov, "The operations on intuitionistic fuzzy values in the framework of Dempster-Shafer theory," Knowledge-Based Systems, 35, 132-143 (2012).

20. J.Q. Wang, R.R. Nie, H.Y. Zhang and X.H. Chen, "Intuitionistic fuzzy multi-criteria decision-making method based on evidential reasoning," Applied Soft Computing, 13, 1823-1831 (2013).

21. S.K. De, R. Biswas and A.R. Roy, "An application of intuitonistic fuzzy sets in medical diagnosis," Fuzzy Sets and Systems, 117(2), 209-213 (2001).

22. H.M. Nehi and H.R. Maleki, "Intuitionistic fuzzy numbers and it's applications in fuzzy optimization problem," Proceeding of the WSEAS international conference on systems, Athens, Greece, 9th, 1-5 (2005).

23. P. Grzegrorzewski, "The hamming distance between intuitionistic fuzzy sets" Proceedings of the IFSA world congress, Istanbul, Turkey, 10th, 35-38 (2003).

24. G. Deschrijver and E.E. Kerre, "On the relationship between some extensions of fuzzy set theory," Fuzzy Sets and Systems, 133, 227-235 (2003).

25. H.J. Zimmermann, "Fuzzy set Theory and Its Applications," Norwell, MA: Kluwer,(1996).

26. Y. Deng, R. Sadiq, W. Jiang and S. Tesfamariam, "Risk analysis in a linguistic environment: A fuzzy evidential reasoning-based approach," Expert Systems with Applications, 38, 15438-15446 (2011).

27. P. Grzegorzewski, "On possible and necessary inclusion of intuitionistic fuzzy sets," Information Sciences, 181, 342-350(2011).

28. P. Grzegorzewski and E. Mrowka, "Trapezoidal approximations of fuzzy numbers," Fuzzy Sets and Systems, 153, 115-135 (2005).

29. S. Tesfamariam, R. Sadiq and H. Najjaran, "Decision making under uncertainty-an example for seismic risk management," Risk Analysis, 30(1), 87-94 (2009).

30. J. Ye, "Expected value method for intuitionistic trapezoidal fuzzy multicriteria decision-making problems," Expert Systems with Applications, 38, 11730-11734 (2011).

31. Y.N.Wang, Y.P. Dai and F.C. Meng, "Similarity measure of intuitionistic trapezoidal fuzzy numbers and its application for medical diagnosis," Proceedings of the Chinese Control Conference (CCC), 32nd, 8567-8571 (2013). 